\title{
Social credibility, social patience and long run inequality.
}

\author{
Christopher Sleet \\ Şevin Yeltekin \\ Carnegie Mellon University Carnegie Mellon and Northwestern Universities
}

Current version: July 2005

\begin{abstract}
This paper considers optimal social insurance in a dynamic moral hazard economy. The existing literature has focussed on environments in which a planner and a population of agents share the same discount factor. A key finding is that agents are then almost surely immiserated; their welfare is driven to its lowest bound. Such immiseration requires a social commitment to treat (almost) everyone arbitrarily badly in the long run. We argue that this is implausible. We establish an equivalence between optimal allocations in two environments: those in which the planner lacks commitment and is constrained to choose socially credible policies and those in which the planner's discount factor exceeds the agents'. We show that immiseration result no longer holds in the second environment. Optimal allocations in this environment are characterised by social mobility and a degree of inequality that it is falling in the planner's discount factor. The credibility constrained economy inherits the no immiseration and social mobility properties.
\end{abstract}




\section{Introduction}

Many recent papers have analysed optimal dynamic contracts in environments with private information frictions. A key area of application is to the design of optimal social insurance schemes. In these a planner or government ${ }^{1}$ insures a population of agents against idiosyncratic, publicly unobserved shocks. In each period, agents report their shocks to the planner and receive a current allocation and a promised future allocation. To induce truthful revelation, it is usually optimal to penalise an agent that reports that he is in need of additional resources today, with a less valuable allocation in the future. Specific applications have considered the optimal design of unemployment and disability insurance, labour and capital taxation. However, a result common to many of these papers is that the optimal social insurance arrangement leads to the eventual immiseration of the agent. Under these arrangements, with probability one, the agent's utility converges to its lowest bound. Expressed in terms of an endogenously evolving Pareto weight, these arrangements imply that the agent's Pareto weight almost surely converges to 0. Discovered initially in a partial equilibrium context by Green (1987) and Thomas and Worrall (1990), this result was extended by Atkeson and Lucas (1992) to general equilibrium settings. In this case, a measure zero set of agents end up with all resources in the economy, while the rest end up with nothing. ${ }^{2}$

The immiseration result has encountered two sorts of criticism. On the one hand some regard it as normatively unappealing, since if an agent is viewed as a dynasty, it implies a very inequitable distribution of consumption across generations. ${ }^{3}$ On the other hand, it requires that the planner or, more generally, society can commit to an allocation that treats almost everyone arbitrarily badly in the long run. This sort of social commitment seems

\footnotetext{
${ }^{1}$ We use the label "planner" throughout. However, we interpret the planner as social device for implementing an allocation, that may itself be subject to incentive problems.

${ }^{2}$ Phelan (1998) links this result to Inada type properties of the agent's utility function.

${ }^{3}$ On this, see Farhi and Werning (2005) and Phelan (2003).
} 
especially demanding and implausible.

In the current paper, motivated by this second sort of criticism, we consider environments in which the planner must satisfy a sequence of credibility constraints. These require that the planner keeps a weighted aggregate of agent utilities above a lower bound. The planner maximises a (possibly different) weighted aggregate of discounted agent utilities; it shares the same discount factor as the agents. Agents receive taste shocks that they privately observe. ${ }^{4}$ Allocations will not be viable, and therefore, will not offer credible incentives for truth-telling, unless they satisfy the credibility constraints. We think of these constraints as capturing the collective ability of agents to resist or avoid reductions in their continuation utilities even if these reductions are implied by a plan to which they, or their ancestors, previously agreed. This ability might stem from social disruption, political pressure of some sort or a straightforward lack of commitment on the part of a benevolent planner. Our model encompasses all of these cases. ${ }^{5}$ It is, therefore, a reduced form for a variety of political economy-type games. We show that this credibility-constrained problem with common discounting is essentially equivalent to a problem without credibility constraints, but with an endogenously determined planner discount factor in excess of the private one.

The link between credibility and social patience described in the previous paragraph provides us with a motivation for studying economies with high social (planner) discount factors. Additionally, it allows us to use analyses of the last sort of economy to establish facts about more complicated environments with credibility constraints. Specifically, we establish several results concerning inequality and social mobility in the high social

\footnotetext{
${ }^{4}$ The taste shock model without credibility constraints was previously analysed by Atkeson and Lucas (1992). Our results extend beyond models with privately observed taste shocks. However, given Atkeson and Lucas's analysis, this type of private information serves as a useful benchmark. We apply our results to a model of unemployment insurance later in the paper.

${ }^{5}$ For an explicit example, see Sleet and Yeltekin (2004). There we consider the case of a benevolent planner who cannot commit. We show that the optimal allocation in a game without planner commitment satisfies credibility constraints.
} 
discount economy and then translate them into equivalent results for the credibility-constrained environment. First, we show that the immiseration result does not generally hold when the planner's discount factor exceeds that of the agents: the zero Pareto weight is no longer an absorbing state. Instead, an agent's endogenously evolving Pareto weight fluctuates around a positive average value. This has immediate implications for social mobility. Second, we give conditions under which the optimal allocation in such settings admits a stationary distribution over endogenously evolving Pareto weights, utilities and consumptions, and conditions under which it admits a strongly ergodic Markov process for Pareto weights. In this last case we can derive a stronger social mobility result that rules out multiple "caste systems", regions of the Pareto weight space in which agents become trapped. ${ }^{6}$ A straightforward economic intuition underpins these results. A planner that is relatively more patient than the agents concentrates the future rewards and punishments necessary to induce truthful reporting into the periods immediately after a report. Since agents attach relatively more weight to these periods, such a concentration of rewards and punishments provides the strongest incentives at least cost to the planner. Consequently, (reported) shocks have transitory, rather than permanent effects on the agent's utility. A relatively high planner discount factor becomes a force for mean reversion of an agent's Pareto weight.

In the context of credibility-constrained economies, we define a stationary credible equilibrium. This consists of an allocation that solves a recursive planner problem with (endogenously) high planner discounting, a stationary distribution over endogenous Pareto weights induced by the policy functions from this problem and a corresponding distribution over agent continuation utilities such that the credibility constraint is just satisfied. We can show that the allocation from this problem solves a planner problem with credibility constraints. Moreover, we can use results from the high planner discount economy to characterise this equilibrium. We obtain further characterisation through numerical examples. In particular, these indicate that as the credibility constraint is tightened, the effective planner discount factor rises. Higher planner discount factors are associated

\footnotetext{
${ }^{6}$ The language is due to Phelan (2003).
} 
with less inequality in the corresponding optimal stationary allocation.

Our paper makes several additional technical contributions. We believe that these will have application beyond the current setting. Most importantly, we adopt the recursive saddle point method of Marcet and Marimon (MM) (1999) rather than the more conventional utility promise method of Green, Thomas and Worrall and others. Our paper, thus, shows how the MM approach can be applied to a private information environment. ${ }^{7}$ The approach leads to an attractive recursive reformulation of the Lagrangian from the planner's choice problem with a very natural economic interpretation. It introduces a "cumulative multiplier", $\zeta_{t}$, that keeps track of an agent's history by aggregating the Lagrange multipliers on the agent's previous incentive-compatibility conditions. This multiplier can be interpreted as an endogenously evolving Pareto-Negishi weight. More precisely, if the planner's (possibly endogenous) discount factor is $\lambda$, and the agent's is $\beta$, then in each of our economies this weight evolves according to:

$$
\zeta_{t+1}=\frac{1}{1+\psi} \zeta_{t}+\left(\frac{\psi}{1+\psi}\right)+\varepsilon_{t}, \quad \text { with } E_{t-1} \varepsilon_{t}=0
$$

where, $\psi=\frac{\lambda-\beta}{\beta}$ and $\varepsilon_{t}$ is a shock. This shock term is derived endogenously from the Lagrange multipliers on the agent's incentive-compatibility conditions. When $\lambda=\beta$ and $\psi=0$, the stochastic process in (1) is a martingale. Under appropriate technical conditions, the martingale convergence theorem holds, the process almost surely converges to 0 and the agent is almost surely immiserated. However, when $\lambda>\beta$ and $\psi>0$, this process tends to mean revert towards 1 . The mean reverting property for the high planner discount case is thus found in a very straightforward and direct way. Our various results on (no) immiseration, social mobility and inequality

\footnotetext{
${ }^{7}$ It is well known, that the MM method is applicable to a narrower category of strictly concave problems, than the utility promise approach. The problems in this paper are in this category. The MM method has largely been applied to Ramsey tax problems or full information problems in which agents cannot commit. Especially in the former case this is often done without any formal verification of its applicability. (See Messner and Pavoni (2004) for a discussion).
} 
stem from this. Moreover, the law of motion (1) is amenable to analysis using a variety of new theorems on Markov processes which we introduce.

The plan for the remainder of the paper is as follows. In Section 2, we start with a basic environment in which the planner has an exogenously higher discount factor than the agents. In Section 3, we break this problem into a family of component planner problems. In each of these a component planner chooses an incentive-compatible allocation to maximise the utility (net of resource cost) of a specific Pareto weighted agent. We show how these component planner problems can be formulated recursively using the agent's Pareto weight. Specifically, we show that the component planner value functions satisfy a sequence of Bellman equations. We give sufficient conditions for the operator associated with these Bellman equations to be a contraction. Since the component planner's value function is typically unbounded, this requires identifying an appropriate weighted norm space on which to search for this value function. In Section 4, we observe that the policy functions from these planner problems are inconsistent with the immiseration of agents. We then turn to a detailed consideration of the planner's problem in constant price economies. We establish conditions under which the Markov process for Pareto-Negishi weights implied by the component planner problems admits a stationary distribution and is strongly ergodic. We interpret these results in terms of both the cross sectional distribution of Pareto-Negishi weights and the social mobility of an individual agent. Section 5 briefly discusses an alternative unemployment insurance environment. In Section 6, we relate the high planner discount economy to an economy with credibility constraints. In particular, we use the planner's Lagrangian to construct an endogenous discounting scheme off of the Lagrange multipliers on the credibility constraints. In Section 7 we explicitly impose a resource constraint on the planner. Section 8 contains numerical results, while Section 9 concludes. 


\section{$1.1 \quad$ Related literature}

The literature on optimal dynamic contracting, and specifically the immiseration result, includes Green (1987), Thomas and Worrall (1990) and Atkeson and Lucas (1992). It has elicited various responses. Atkeson and Lucas (1995) consider a model in which the planner must respect an exogenous lower bound on each agent's utility. They motivate this by appealing to considerations of equality. Phelan (1995) imposes a lack of commitment friction on agents. They can exit a social insurance arrangement at a cost and enter into another arrangement. His model can be interpreted as one in which the planner is constrained in its ability to enforce social arrangements.

Phelan (2003) and Farhi and Werning (2005) also consider environments in which the planner's discount factor exceeds the agents. Phelan analyses the case in which the planner's discount factor equals 1 . He shows that the immiseration result is overturned in this case. Farhi and Werning is most related to us. Like us, they consider environments in which the planner's discount factor is between that of the agent and one. They find conditions under which a stationary distribution over agent utilities exists. Their formulation of the problem is different from ours, it relies upon the utility promise keeping rather than the MM approach, as is their method of proof. Moreover, they take their problem to a different (and very interesting) set of applications; they consider how their optimal allocations could be implemented with estate taxation, while we consider the connection between high planner discount factors and credibility.

\section{Environment}

In this section we describe the environment and introduce notation.

Our economy is inhabited by a continuum of infinitely-lived agents and a planner. Each agent has preferences 
over stochastic sequences for consumption $\left\{c_{t}\right\}_{t=0}^{\infty}$ of the form:

$$
E\left[\sum_{t=0}^{\infty} \beta^{t} \theta_{t} u\left(c_{t}\right)\right] .
$$

Here $\beta \in(0,1)$ denotes the agent's discount factor, while $\theta_{t} \in \Theta$ is a period $t$ idiosyncratic taste shock that is privately observed by the agent. Shocks are assumed to satisfy:

Assumption 1 1) $\Theta=\left\{\widehat{\theta}_{k}\right\}_{k=1}^{K}, \widehat{\theta}_{k+1}>\widehat{\theta}_{k}>0$;2) $\left\{\theta_{t}\right\}$ is independently and identically distributed over time and agents with distribution $\pi .^{8}$

Define $p_{k}:=\pi\left(\widehat{\theta}_{k}\right) / \pi\left(\widehat{\theta}_{k-1}\right)$ and let $\theta^{t}:=\left\{\theta_{0}, \theta_{1}, \ldots, \theta_{t}\right\} \in \Theta^{t+1}$ denote a $t$-period history of shocks. Let $\pi^{t}$ denote the corresponding probability distribution. The utility function $u$ is assumed to satisfy:

Assumption 2 1) $u: \mathbb{R}_{+} \rightarrow D \subseteq \mathbb{R} \cup\{-\infty\}$ is a strictly increasing and strictly concave function. It is continuously differentiable on $(0, \infty)$; 2) $\lim _{c \rightarrow \infty} u^{\prime}(c)=0$; 3) Either $\sup D<\infty$ or $\inf D>-\infty$.

It is convenient to work with allocations of utility rather than consumption. Formally, we define a utility allocation to be a sequence of functions $\alpha=\left\{u_{t}\right\}_{t=0}^{\infty}$, where $u_{t}: \Theta^{t+1} \rightarrow D$ gives the utility from consumption obtained by an agent at date $t$. The associated consumption allocation can then be recovered using the inverse of $u, C: D \rightarrow \mathbb{R}_{+}$. For $t>0$, let $\alpha\left(\theta^{t-1}\right):=\left\{u_{t+s}\left(\theta^{t-1}, \cdot\right)\right\}_{s=0}^{\infty}$ denote the continuation of $\alpha$ after the history $\theta^{t-1}$. For $t=0$, set $\alpha\left(\theta^{-1}\right):=\alpha$. Let $A$ denote the set of utility allocations.

The planner assigns a utility allocation to each agent in period 0. Since she cannot observe an agent's shock history, the planner cannot condition the agent's utility award directly upon this. Instead, she conditions it upon histories of shock reports given by the agent. The reporting behaviour of agents is described by a reporting policy $\delta=\left\{\delta_{t}\right\}_{t=0}^{\infty}$, where $\delta_{t}: \Theta^{t} \rightarrow \Theta$ gives the $t$-th period report of an agent conditional on the agent's past history of shocks. For each $t$ and all $\theta^{t}$, let $\delta^{t}\left(\theta^{t}\right)=\left(\delta^{t-1}\left(\theta^{t-1}\right), \delta_{t}\left(\theta^{t}\right)\right)$ denote the corresponding history of reports implied

\footnotetext{
${ }^{8}$ We also interpret $\pi\left(\widehat{\theta}_{k}\right)$ as the fraction of agents receiving the shock $\widehat{\theta}_{k}$. In doing so we rely on the argument of Judd (1985).
} 
by $\delta$. Agents choose a reporting policy in period 0 . If the planner assigns a (report-contingent) utility allocation $\alpha=\left\{u_{t}\right\}_{t=0}^{\infty}$ to an agent and the agent chooses reporting policy $\delta$, then the realised allocation of the agent is $\alpha \circ \delta:=\left\{u_{t}\left(\delta^{t}(\cdot)\right)\right\}_{t=0}^{\infty}$. Invoking the revelation principle, we can restrict attention to incentive-compatible utility allocations that induce the agent to be truthful. Formally, $\alpha$ is said to be incentive-compatible if:

$$
\forall \delta: U_{0}(\alpha) \geq U_{0}(\alpha \circ \delta)
$$

where for $t \geq 0, U_{t}\left(\alpha\left(\theta^{t-1}\right)\right):=E_{\theta^{t-1}} \sum_{s=0}^{\infty} \beta^{s} \theta_{t+s} u_{t+s}\left(\theta^{t+s}\right)$ is the payoff to the agent from the continuation allocation $\alpha\left(\theta^{t-1}\right)=\left\{u_{t+s}\left(\theta^{t-1}, \cdot\right)\right\}_{s=0}^{\infty}$.

The planner has a discount factor $\lambda \in[\beta, 1)$. Each agent in the population is indexed by a pair of numbers $\left(\zeta_{0}, \gamma\right) \in Z \times Z$, where $Z=\mathbb{R}_{+}$if the agent's utility function is unbounded below, and $Z=\mathbb{R}$ otherwise. The $\left(\zeta_{0}, \gamma\right)$-th agent's utility allocation is denoted $\alpha^{\left(\zeta_{0}, \gamma\right)}=\left\{u_{t}^{\left(\zeta_{0}, \gamma\right)}\right\}_{t=0}^{\infty}$. We assume that each $\alpha^{(\cdot, \cdot)}$ is Borel measurable. The planner weights the per period utility of the $\left(\zeta_{0}, \gamma\right)$-th agent using the generalised Pareto-Negishi weighting scheme $\left\{\gamma_{t}\left(\zeta_{0}, \gamma\right)\right\}_{t=0}^{\infty}$ where

$$
\gamma_{t}\left(\zeta_{0}, \gamma\right)=\gamma+\left(\frac{1}{1+\psi}\right)^{t}\left(\zeta_{0}-\gamma\right)
$$

and $\psi=\frac{\lambda-\beta}{\beta}$. Since $\gamma_{0}\left(\zeta_{0}, \gamma\right)=\zeta_{0}$ and $\lim _{t \rightarrow \infty} \gamma_{t}\left(\zeta_{0}, \gamma\right)=\gamma$, the parameters $\left(\zeta_{0}, \gamma\right)$ may be interpreted as the agent's initial and long run Pareto weight. Intuitively, we may imagine that events prior to period 0 have caused the agent to be temporarily rewarded (or punished) with an initial Pareto-Negishi weight above (or below) its long run level. If $\zeta_{0}=\gamma($ or $\lambda=\beta)$, then $\gamma_{t}\left(\zeta_{0}, \gamma\right)$ is constant for all $t$. Thus, our formulation accommodates the more standard constant Pareto weighting scheme as a special case. Generalising the scheme in this way anticipates our later recursive formulation and allows us to be more flexible in our treatment of the initial period. We assume a cross sectional distribution over $\gamma$ values given by $\Psi_{0}$ and a distribution over $\zeta_{0}$ values given by $\Phi_{0}$. 
The planner faces a sequence of intertemporal resource prices $\left\{\lambda^{t} q_{t}\right\}_{t=0}^{\infty}$, where each $q_{t} \in[\underline{q}, \bar{q}], 0<\underline{q}<\bar{q}<\infty$, so that her net-of-cost objective is given by:

$$
\begin{aligned}
W\left(\left\{\alpha^{\left(\zeta_{0}, \gamma\right)}\right\} ; \Psi_{0}, \Phi_{0},\left\{q_{t}\right\}_{t=0}^{\infty}\right)= & \int_{Z} \int_{Z} \sum_{t=0}^{\infty} \lambda^{t} \gamma_{t}\left(\zeta_{0}, \gamma\right) \sum_{\theta^{t} \in \Theta^{t+1}} \theta_{t} u_{t}^{\left(\zeta_{0}, \gamma\right)}\left(\theta^{t}\right) \pi^{t}\left(\theta^{t}\right) \Phi_{0}\left(d \zeta_{0}\right) \Psi_{0}(d \gamma) \\
& -\int_{Z} \int_{Z} \sum_{t=0}^{\infty} \lambda^{t} q_{t} \sum_{\theta^{t} \in \Theta^{t+1}} C\left(u_{t}^{\left(\zeta_{0}, \gamma\right)}\left(\theta^{t}\right)\right) \pi^{t}\left(\theta^{t}\right) \Phi_{0}\left(d \zeta_{0}\right) \Psi_{0}(d \gamma) .
\end{aligned}
$$

For the moment, we take the price sequence as a parameter. However, this formulation of preferences accommodates a variety of resource constraints upon the planner. For example, the sequence $\left\{\lambda^{t} q_{t}\right\}$ may represent a sequence of optimal shadow prices from a problem in which the planner faces a per period aggregate resource constraint. We elaborate on these possibilities in Section 7. The planner's problem is then given by:

$$
\begin{aligned}
& \sup _{\left\{\alpha^{\left(\zeta_{0}, \gamma\right)} \in A\right\}} W\left(\left\{\alpha^{\left(\zeta_{0}, \gamma\right)}\right\} ; \Psi_{0}, \Phi_{0},\left\{q_{t}\right\}_{t=0}^{\infty}\right) \\
& \text { s.t. for } \Phi_{0} \text {-a.e. } \zeta_{0}, \Psi_{0} \text {-a.e. } \gamma \text {, and } \forall \delta: U_{0}\left(\alpha^{\left(\zeta_{0}, \gamma\right)}\right) \geq U_{0}\left(\alpha^{\left(\zeta_{0}, \gamma\right)} \circ \delta\right) .
\end{aligned}
$$

\section{The component planner's problem}

We now turn to the analysis of the planner's problem (6). We proceed in the following steps. First, we define a relaxed planner's problem that incorporates a less restrictive set of "temporary" incentive constraints. Then we disaggregate this problem into a family of component planner problems in which a single planner deals with a single agent. Finally, we obtain a recursive formulation of these component planner's problems using the techniques of Marcet and Marimon. The first two steps are fairly standard in the dynamic contracting literature. The third is less so in models with private information. To date, application of the Marcet and Marimon approach has mainly been confined to Ramsey tax problems or to problems without agent commitment. We extend the approach to accommodate our private information environment. 
Given an allocation $\alpha$, we define the temporary incentive constraints by

$$
\forall t, \theta^{t-1}, k, j: \Delta U_{t}\left(\alpha\left(\theta^{t-1}\right), \widehat{\theta}_{k}, \widehat{\theta}_{j}\right) \geq 0
$$

where $\Delta U_{t}\left(\alpha\left(\theta^{t-1}\right), \widehat{\theta}_{k}, \widehat{\theta}_{j}\right):=\widehat{\theta}_{k} u_{t}\left(\theta^{t-1}, \widehat{\theta}_{k}\right)+\beta U_{t+1}\left(\alpha\left(\theta^{t-1}, \widehat{\theta}_{k}\right)\right)-\widehat{\theta}_{k} u_{t}\left(\theta^{t-1}, \widehat{\theta}_{j}\right)-\beta U_{t+1}\left(\alpha\left(\theta^{t-1}, \widehat{\theta}_{j}\right)\right)$ gives the difference in payoffs obtained by an agent with current shock $\widehat{\theta}_{k}$ from the allocations $\left\{u_{t}\left(\theta^{t-1}, \widehat{\theta}_{k}\right), \alpha\left(\theta^{t-1}, \widehat{\theta}_{k}\right)\right\}$ and $\left\{u_{t}\left(\theta^{t-1}, \widehat{\theta}_{j}\right), \alpha\left(\theta^{t-1}, \widehat{\theta}_{j}\right)\right\} .^{9}$ The constraints (7) require that after each history of shocks, the agent is better off truthfully reporting her state, rather than lying and being truthful thereafter. Our earlier incentive constraint (3) clearly implies (7). Conversely, by a well known result (e.g. Atkeson and Lucas (1992)), an allocation satisfying (7), and the limiting conditions

$$
\forall \theta^{\infty}, \lim _{t \rightarrow \infty} \beta^{t} \sum_{s=0}^{\infty} \beta^{s} \theta_{t+s} u_{t+s}\left(\theta^{t+s}\right)=0
$$

satisfies (3) as well. In the sequel we will consider planner's problems subject only to temporary incentive constraints (7). If $u$ is bounded, it is immediate that all allocations satisfy (8) and so allocations satisfying (7) are incentive compatible. Similarly, if a solution to the planner's problem with temporary incentive constraints exists, and if it ensures that the agent's continuation payoffs are uniformly bounded, then it satisfies (8) and is incentive compatible. ${ }^{10}$ We further relax the planner's problem by imposing only the local upwards temporary incentive constraints:

$$
\forall t, \theta^{t-1}, k \in\{0, \ldots, K-1\}: \Delta U_{t}\left(\alpha\left(\theta^{t-1}\right), \widehat{\theta}_{k}, \widehat{\theta}_{k+1}\right) \geq 0
$$

In the appendix we verify that a solution to the relaxed planner's problem with only local upwards constraints

\footnotetext{
${ }^{9}$ We use the convention $\infty-\infty=-\infty+\infty=0$ to ensure that $\Delta U_{t}\left(\alpha\left(\theta^{t-1}\right), \widehat{\theta}_{k}, \widehat{\theta}_{j}\right)$ is always well defined.

${ }^{10}$ The constraints (8) are demanding and it is difficult to check directly that the set of allocations satisfying these and the temporary incentive compatibility constraints is compact. The strategy of relaxing the problem is standard (Atkeson and Lucas (1992)).
} 
satisfies all temporary incentive compatibility constraints. Formally, our relaxed problem is:

$$
\begin{aligned}
& \sup _{\left\{\alpha^{\left(\zeta_{0}, \gamma\right)} \in A\right\}} W\left(\left\{\alpha^{\left(\zeta_{0}, \gamma\right)}\right\} ; \Psi_{0}, \Phi_{0},\left\{q_{t}\right\}_{t=0}^{\infty}\right) \\
& \text { s.t. for } \Phi_{0} \text {-a.e. } \zeta_{0}, \Psi_{0} \text {-a.e. } \gamma \text {, and } \forall t, \theta^{t-1}, k<K \quad \lambda^{t} \Delta U_{t}\left(\alpha^{\left(\zeta_{0}, \gamma\right)}\left(\theta^{t-1}\right), \widehat{\theta}_{k}, \widehat{\theta}_{k+1}\right) \geq 0 .
\end{aligned}
$$

To solve (10), we disaggregate it into a collection of component planner problems. In each of these a component planner maximises the net-of-cost utility for a specific $\left(\zeta_{0}, \gamma\right)$-weighted agent. To economise on notation, we restrict attention to the population of agents sharing a common $\gamma$ and drop the explicit indexing of objects by $\gamma$. The $r$-th period continuation component planner problem is then:

$$
\begin{gathered}
V_{r}^{*}\left(\zeta_{r}\right)=\sup _{\alpha \in A} \sum_{t=0}^{\infty} \lambda^{t} \gamma_{t}\left(\zeta_{r}\right) \sum_{\theta^{t} \in \Theta^{t+1}} \theta_{t} u_{t}\left(\theta^{t}\right) \pi^{t}\left(\theta^{t}\right)-\sum_{t=0}^{\infty} \lambda^{t} q_{r+t} \sum_{\theta^{t} \in \Theta^{t+1}} C\left(u_{t}\left(\theta^{t}\right)\right) \pi^{t}\left(\theta^{t}\right) \\
\text { s.t. } \forall t, \theta^{t-1}, k<K \quad \lambda^{t} \Delta U_{t}\left(\alpha\left(\theta^{t-1}\right), \widehat{\theta}_{k}, \widehat{\theta}_{k+1}\right) \geq 0 .
\end{gathered}
$$

In this continuation problem, the component planner enters period $r$ with current Pareto-Negishi weight $\zeta_{r}$. The planner faces the price sequence $\left\{q_{r+t}\right\}_{t=0}^{\infty}$ and chooses a utility allocation $\left\{u_{t}\right\}_{t=0}^{\infty}$ to maximise her payoff. We reformulate this problem recursively using the method of Marcet and Marimon (1999). Before giving the details, we present a heuristic overview of our formulation.

\subsection{Applying the Marcet-Marimon approach: A heuristic overview}

In this subsection, we write down and manipulate a Lagrangian for the component planner problem (11). We use the Lagrangian to convert this problem into one without incentive constraints, but with an endogenously evolving Pareto-Negishi weight. After low shock reports this weight is increased, after high shock reports it is reduced, capturing the need to offer future rewards for the first type of report and future punishments for the second. We proceed informally and relegate detailed proofs to later in the section.

To keep matters as simple as possible, suppose that the number of shocks, $K$, equals 2 . In this case, there 
is only one upwards incentive constraint after each history $\theta^{t-1}$. We denote its multiplier by $\eta_{t}\left(\theta^{t-1}, \widehat{\theta}_{1}\right)$. A Lagrangian for problem (11) with $r=0$ can then be written as:

$$
\begin{aligned}
\mathcal{L}= & \sum_{t=0}^{\infty} \lambda^{t} \gamma_{t}\left(\zeta_{0}\right) \sum_{\theta^{t} \in \Theta^{t+1}} \theta_{t} u_{t}\left(\theta^{t}\right) \pi^{t}\left(\theta^{t}\right)-\sum_{t=0}^{\infty} \lambda^{t} q_{t} \sum_{\theta^{t} \in \Theta^{t+1}} C\left(u_{t}\left(\theta^{t}\right)\right) \pi^{t}\left(\theta^{t}\right) \\
& +\sum_{t=0}^{\infty} \lambda^{t} \sum_{\theta^{t-1} \in \Theta^{t}} \eta_{t}\left(\theta^{t-1}, \widehat{\theta}_{1}\right) \Delta U_{t}\left(\alpha\left(\theta^{t-1}\right), \widehat{\theta}_{1}, \widehat{\theta}_{2}\right) \pi^{t-1}\left(\theta^{t-1}\right) \pi\left(\widehat{\theta}_{1}\right) .
\end{aligned}
$$

Suppose that a solution to the component planner's problem and a corresponding optimal multiplier sequence $\left\{\eta_{t}^{*}\left(\theta^{t-1}, \widehat{\theta}_{1}\right)\right\}_{t=0}^{\infty}$ attain the saddle point of this Lagrangian. For notational convenience, define for each $\theta^{t-1}$, $\eta_{t}^{*}\left(\theta^{t-1}, \widehat{\theta}_{2}\right)=\eta_{t}^{*}\left(\theta^{t-1}, \widehat{\theta}_{0}\right)=0$. Also, define $\varsigma: \Theta \rightarrow \Theta$ pointwise by $\varsigma\left(\widehat{\theta}_{1}\right)=\widehat{\theta}_{2}$ and $\varsigma\left(\widehat{\theta}_{2}\right)=\widehat{\theta}_{1}$. Rearranging (12) evaluated at the optimal multiplier sequence, and using this new notation, we obtain:

$$
\sum_{t=0}^{\infty} \sum_{\theta^{t} \in \Theta^{t+1}} \lambda^{t} \pi\left(\theta^{t}\right)\left\{\left[\zeta_{t}^{*}\left(\theta^{t-1}\right)+\left(\eta_{t}^{*}\left(\theta^{t}\right)-\eta_{t}^{*}\left(\theta^{t-1}, \varsigma\left(\theta_{t}\right)\right) p_{1} \frac{\varsigma\left(\theta_{t}\right)}{\theta_{t}}\right)\right] \theta_{t} u_{t}\left(\theta^{t}\right)-q_{t} C\left(u_{t}\left(\theta^{t}\right)\right)\right\}
$$

where

$$
\zeta_{t}^{*}\left(\theta^{t-1}\right)=\gamma_{t}\left(\zeta_{0}, \gamma\right)+\sum_{s=0}^{t}\left(\frac{\beta}{\lambda}\right)^{t-s}\left\{\eta_{s}^{*}\left(\theta^{s}\right)-\eta_{s}^{*}\left(\theta^{s-1}, \varsigma\left(\theta_{s}\right)\right) p_{1}\right\}
$$

It follows that the solution to the component planner's problem maximises (13). Such a maximisation corresponds to a planner's problem without incentive constraints, but with an endogenously evolving Pareto-Negishi weight $\left\{\zeta_{t}^{*}\right\}$. As (14) indicates this weight augments the agent's original Pareto weight with a term that depends on lagged incentive multipliers. Inspection of (14) reveals that $\left\{\zeta_{t}^{*}\right\}$ evolves in a recursive fashion according to:

$$
\zeta_{t+1}^{*}\left(\theta^{t}\right)=\frac{\psi}{1+\psi} \gamma+\frac{1}{1+\psi} \zeta_{t}^{*}\left(\theta^{t-1}\right)+\frac{1}{1+\psi} \varepsilon_{t}^{*}\left(\theta^{t}\right)
$$

where $\psi=\frac{\lambda-\beta}{\beta}>0$ and $\varepsilon_{t}^{*}\left(\theta^{t}\right)=\eta_{t}^{*}\left(\theta^{t}\right)-\eta_{t}^{*}\left(\theta^{t-1}, \varsigma\left(\theta_{t}\right)\right) p_{1}$. Now, $\varepsilon_{t}^{*}\left(\theta^{t-1}, \widehat{\theta}_{1}\right)=\eta_{t}^{*}\left(\theta^{t-1}, \widehat{\theta}_{1}\right)$, while $\varepsilon_{t}^{*}\left(\theta^{t-1}, \widehat{\theta}_{2}\right)=$ $-\eta_{t}^{*}\left(\theta^{t-1}, \widehat{\theta}_{1}\right)$, so that $\sum_{k=1}^{2} \varepsilon_{t}^{*}\left(\theta^{t-1}, \widehat{\theta}_{k}\right) \pi\left(\widehat{\theta}_{k}\right)=0$. Consequently, we may think of each $\varepsilon_{t}^{*}$ as an "incentive shock" that perturbs the endogenous Pareto-Negishi weight. If the agent receives the low shock at $t$, then $\varepsilon_{t}^{*}\left(\theta^{t-1}, \widehat{\theta}_{1}\right)=$ $\eta_{t}^{*}\left(\theta^{t-1}, \widehat{\theta}_{1}\right)>0$ and the Pareto-Negishi weight $\zeta_{t+1}^{*}\left(\theta^{t}\right)$ is relatively increased. Intuitively, to induce an agent to 
truthfully report the low shock value $\widehat{\theta}_{1}$ in period $t$ (and receive low consumption in that period), the planner must reward the agent with higher utility in the future. She does this by raising the agent's Pareto-Negishi weight at $t+1$. Conversely, if the agent receives the high shock, then $\varepsilon_{t}^{*}\left(\theta^{t-1}, \widehat{\theta}_{2}\right)=-\eta_{t}^{*}\left(\theta^{t-1}, \widehat{\theta}_{1}\right) p_{1}<0$ and the agent's Pareto-Negishi weight at $t+1$ falls.

When $\lambda=\beta$, then $\psi=0,(15)$ reduces to:

$$
\zeta_{t+1}^{*}\left(\theta^{t}\right)=\zeta_{t}^{*}\left(\theta^{t-1}\right)+\varepsilon_{t}^{*}\left(\theta^{t}\right)
$$

and $\left\{\zeta_{t}^{*}\right\}$ is a martingale. In Sleet and Yeltekin (2004), we use this observation and the martingale convergence theorem to derive an immiseration result for economies in which the planner discounts at the same rate as the agents. More generally, when $\lambda>\beta, \psi>0$ and the coefficient in front of $\zeta_{t}^{*}$ in (15) is less than one in absolute value. Thus, the process for Pareto-Negishi weights has a tendency to mean revert. Intuitively, when $\lambda>\beta$ the agent attaches greater relative value to utility received in the shorter run than the planner. Consequently, the planner concentrates rewards and punishments for a report in the periods immediately after the report.

\subsection{Analysis of the Component planner's problem}

Our goal in this section is to obtain a recursive formulation of the component planner's problem that uses endogenously evolving Pareto-Negishi weights to keep track of histories. We will now assume $\lambda>\beta .{ }^{11}$ As a preliminary, we first confirm that a solution exists to the component planner's problem (11) and then establish several useful facts about the value function $V_{r}^{*}$. The proof of existence is complicated by the fact that we only impose boundedness above or below (but not both) on the agent's utility function.

Proposition 1 There exists a solution $\left\{u_{r+t}^{*}\left(\zeta_{r}\right)\right\}_{t=0}^{\infty}$ to the $r$-th period component planner's problem (11).

Proof: See Appendix A.

\footnotetext{
${ }^{11}$ For analysis of the $\lambda=\beta$ case, see Sleet and Yeltekin (2004).
} 
For $\zeta \in Z$, define the allocation $\underline{\alpha}(\zeta)$ by setting, $\forall t, \theta^{t}, u_{t}\left(\theta^{t}\right)=\arg \sup _{u \in D} \gamma_{t}(\zeta) E[\theta] u-\bar{q} C(u)$. Denote its payoff to the component planner at the constant price sequence $\bar{q}$ by $\underline{V}(\zeta)>-\infty .{ }^{12} \underline{\alpha}(\zeta)$ offers the agent no insurance. It is clearly incentive compatible and feasible for problem (11) since it is independent of any reports given by the agent. This coupled with the fact that for all $t, q_{t} \leq \bar{q}$ implies, for all $r$ and $\zeta, V_{r}(\zeta) \geq \underline{V}(\zeta)$. Similarly, define $\bar{\alpha}(\zeta)$ by setting, $\forall t, \theta^{t}, u_{t}\left(\theta^{t}\right)=\arg \sup _{u \in D} \gamma_{t}(\zeta) \theta_{t} u-\underline{q} C(u)$. Denote its payoff evaluated at the constant price sequence $\underline{q}$ by $\bar{V}(\zeta)$. Since $\bar{\alpha}(\zeta)$ offers the agent full insurance and for all $t, \underline{q} \leq q_{t}$, then for all $r$ and $\zeta, \bar{V}(\zeta) \geq V_{r}^{*}(\zeta)$. We collect this and other facts about $V_{r}^{*}$ below.

Lemma $1 V_{r}^{*}$ is convex, continuous and differentiable on int $Z$. It is bounded above by $\bar{V}$ and below by $\underline{V}$. If $\inf D \geq 0, V_{r}^{*}$ is increasing; if $\sup D \leq 0, V_{r}^{*}$ is decreasing.

\section{Proof: See Appendix B.}

In the proof of Proposition 1, we establish that any candidate optimal plan satisfies $\sum_{t=0}^{\infty} \lambda^{t} \sum_{\theta^{t}} \theta_{t}\left|u_{t}\left(\theta^{t}\right)\right| \pi^{t}\left(\theta^{t}\right)$ $<\infty$ (which, of course, implies that $\sum_{t=0}^{\infty} \beta^{t} \sum_{\theta^{t}} \theta_{t}\left|u_{t}\left(\theta^{t}\right)\right| \pi^{t}\left(\theta^{t}\right)<\infty$ ). Moreover, given these inequalities and the bounds in Lemma 1 , we have that $\sum_{t=0}^{\infty} \lambda^{t}\left|C\left(u_{t}\left(\theta^{t}\right)\right)\right| \pi^{t}\left(\theta^{t}\right)<\infty$. Let $\Omega=\left\{\alpha: \sum_{t=0}^{\infty} \lambda^{t}\right.$ $\sum_{\theta^{t}} \theta_{t}\left|u_{t}\left(\theta^{t}\right)\right| \pi^{t}\left(\theta^{t}\right)<\infty$ and $\left.\sum_{t=0}^{\infty} \lambda^{t}\left|C\left(u_{t}\left(\theta^{t}\right)\right)\right| \pi^{t}\left(\theta^{t}\right)<\infty\right\}$. Associated with each problem (11) is the Lagrangian $\mathcal{L}_{r}\left(\cdot ; \zeta_{r}\right): \mathbb{R}_{+}^{K-1} \times \Omega \rightarrow \mathbb{R}:$

$$
\begin{aligned}
\mathcal{L}_{r}\left(\eta, \alpha ; \zeta_{r}\right)= & \sum_{t=0}^{\infty} \lambda^{t} \gamma_{t}\left(\zeta_{r}\right) \sum_{\theta^{t} \in \Theta^{t+1}} \theta_{t} u_{t}\left(\theta^{t}\right) \pi^{t}\left(\theta^{t}\right)-\sum_{t=0}^{\infty} \lambda^{t} q_{r+t} \sum_{\theta^{t} \in \Theta^{t+1}} C\left(u_{t}\left(\theta^{t}\right)\right) \pi^{t}\left(\theta^{t}\right) \\
& +\sum_{k=1}^{K-1} \eta\left(\widehat{\theta}_{k}\right) \Delta U_{0}\left(\alpha, \widehat{\theta}_{k}, \widehat{\theta}_{k+1}\right) \pi\left(\widehat{\theta}_{k}\right) .
\end{aligned}
$$

This Lagrangian incorporates only the initial period incentive constraints, assigning them the Lagrange multiplier $\eta \in \mathbb{R}_{+}^{K-1}$. It is well defined given the restriction of allocations to $\Omega$. Let $\Omega_{j} \subset \Omega$ denote the set of utility

\footnotetext{
${ }^{12}$ If $\zeta=0$ and $\inf D=-\infty$, then $u_{0}^{*}=-\infty$. In this case, we assign $\zeta u_{0}^{*}$ the value of 0 .
} 
allocations in $\Omega$ that satisfy the local upward incentive compatibility constraints from period $j$ onwards. The following argument relates the Lagrangian $\mathcal{L}_{r}$ to $V_{r}^{*}$ and establishes the existence of an optimising multiplier.

Proposition $2 V_{r}^{*}$ and $\mathcal{L}_{r}$ satisfy for each $\zeta \in Z$,

$$
V_{r}^{*}(\zeta)=\inf _{\eta \in \mathbb{R}_{+}^{K-1}} \sup _{\alpha \in \Omega_{1}} \mathcal{L}_{r}(\eta, \alpha ; \zeta)
$$

Additionally, there exists an $\eta_{r}^{*}(\zeta) \in \mathbb{R}_{+}^{K-1}$ such that $\left(\eta_{r}^{*}(\zeta),\left\{u_{r+t}^{*}(\zeta)\right\}_{t=0}^{\infty}\right)$ attains the saddle point in (18).

Proof: The result follows from Luenberger, Theorem 1 (p. 217) and Corollary 1 (p. 219) and Proposition 1 of this paper.

Gathering together common $u_{t}$ terms in (17) and using the definition of $\gamma_{t}$, we obtain: ${ }^{13}$

$$
\begin{aligned}
& V_{r}^{*}\left(\zeta_{r}\right)=\sup _{\alpha \in \Omega_{1}} \inf _{\substack{\eta \in \mathbb{R}_{+}^{K+1} \\
\eta\left(\hat{\theta}_{0}\right)=\eta\left(\widehat{\theta}_{K}\right)=0}} \sum_{k=1}^{K}\left[\left(\zeta_{r}+\eta\left(\widehat{\theta}_{k}\right)-\eta\left(\widehat{\theta}_{k-1}\right) \frac{\widehat{\theta}_{k-1}}{\widehat{\theta}_{k}} p_{k-1}\right) \widehat{\theta}_{k} u_{0}\left(\widehat{\theta}_{k}\right)-q_{r} C\left(u_{0}\left(\widehat{\theta}_{k}\right)\right)\right. \\
& \left.\quad+\lambda \sum_{t=0}^{\infty} \sum_{\theta^{t} \in \Theta^{t+1}} \lambda^{t}\left[\gamma_{t}\left(\zeta_{r+1}\left(\zeta_{r}, \widehat{\theta}_{k}\right)\right) \theta_{t} u_{t+1}\left(\widehat{\theta}_{k}, \theta^{t}\right)-q_{r+t+1} C\left(u_{t+1}\left(\widehat{\theta}_{k}, \theta^{t}\right)\right)\right] \pi^{t}\left(\theta^{t}\right)\right] \pi\left(\widehat{\theta}_{k}\right),
\end{aligned}
$$

where

$$
\zeta_{r+1}\left(\zeta_{r}, \widehat{\theta}_{k}\right):=\frac{1}{1+\psi} \zeta_{r}+\left(\frac{\psi}{1+\psi}\right) \gamma+\frac{1}{1+\psi}\left[\eta\left(\widehat{\theta}_{k}\right)-\eta\left(\widehat{\theta}_{k-1}\right) p_{k-1}\right]
$$

We now turn to the main result of this section: we establish that the functions $\left\{V_{r}^{*}\right\}_{r=0}^{\infty}$ satisfy a sequence of Bellman equations. In these equations, the Lagrange multipliers from the current incentive constraints $\eta$ appear as choice variables, while the endogenous Pareto-Negishi weight $\zeta$ acts as a state variable. The utility variables are completely eliminated.

We sketch the derivation of the Bellman equations next. First, by the Lagrange duality theorem (Luenberger (1969), Theorem 1, p. 224), we can break the saddle point problem in (19) into two sequential problems. In the

\footnotetext{
${ }^{13}$ Such rearrangements are possible given the restriction to allocations in $\Omega$.
} 
second of these, we maximise over allocations given a multiplier choice, in the first we minimise over multipliers. Since the initial period incentive constraint has been brought into the Lagrangian, the maximisation step involves choosing a family of allocations, $\forall k,\left(u_{0}\left(\widehat{\theta}_{k}\right), \alpha\left(\widehat{\theta}_{k}\right)\right) \in D \times \Omega_{0}$ to solve:

$$
\begin{aligned}
& \sum_{k=1}^{K}\left[\sup _{u_{0}\left(\widehat{\theta}_{k}\right) \in D}\left[\zeta_{r}+\eta\left(\widehat{\theta}_{k}\right)-\eta\left(\widehat{\theta}_{k-1}\right) \frac{\widehat{\theta}_{k-1}}{\widehat{\theta}_{k}} p_{k-1}\right] \widehat{\theta}_{k} u_{0}\left(\widehat{\theta}_{k}\right)-q_{r} C\left(u_{0}\left(\widehat{\theta}_{k}\right)\right)\right. \\
& \left.\quad+\lambda \sup _{\alpha\left(\widehat{\theta}_{k}\right) \in \Omega_{0}} \sum_{t=0}^{\infty} \sum_{\theta^{t} \in \Theta^{t+1}} \lambda^{t}\left[\gamma_{t}\left(\zeta_{r+1}\left(\zeta_{r}, \widehat{\theta}_{k}\right)\right) \theta_{t} u_{t+1}\left(\widehat{\theta}_{k}, \theta^{t}\right)-q_{r+t+1} C\left(u_{t+1}\left(\widehat{\theta}_{k}, \theta^{t}\right)\right)\right] \pi^{t}\left(\theta^{t}\right)\right] \pi\left(\widehat{\theta}_{k}\right) .
\end{aligned}
$$

Define the indirect cost function $W$ by $W\left(\rho ; q_{r}\right)=\sup _{u \in D} \rho u-q_{r} C(u)$. Using (20), the definition of $W$ and that of the value function $V_{r+1}^{*}$, the minimisation step may then be written as:

$$
V_{r}^{*}\left(\zeta_{r}\right)=\inf _{\substack{\eta \in \mathbb{R}_{+}^{K+1}, \eta\left(\hat{\theta}_{0}\right)=\eta\left(\widehat{\theta}_{K}\right)=0}} \sum_{k=1}^{K}\left[W\left(\left[\zeta_{r}+\eta\left(\widehat{\theta}_{k}\right)-\eta\left(\widehat{\theta}_{k-1}\right) \frac{\widehat{\theta}_{k-1}}{\widehat{\theta}_{k}} p_{k-1}\right] \widehat{\theta}_{k} ; q_{r}\right)+\lambda V_{r+1}^{*}\left(\zeta_{r+1}\left(\zeta_{r}, \widehat{\theta}_{k}\right)\right)\right] \pi\left(\widehat{\theta}_{k}\right) .
$$

It follows that the functions $\left\{V_{r}^{*}\right\}$ satisfy a sequence of Bellman equations.

In the subsequent analysis, it is convenient to find a compact constraint correspondence for the multiplier variables $\eta$ in the minimisation step. This leads us to slightly modify the Bellman equation in (21). In Lemma B2 in the appendix, we find a collection of non-binding constraints on the optimal multiplier choices from (18). We use these to construct a compact-valued, non-binding constraint correspondence which we incorporate into the minimisation step. Specifically, we define $\Lambda: Z \rightarrow \mathbb{R}_{+}^{K+1}$ pointwise as follows. If $\zeta<0, \Lambda(\zeta)=\{0\}$, otherwise:

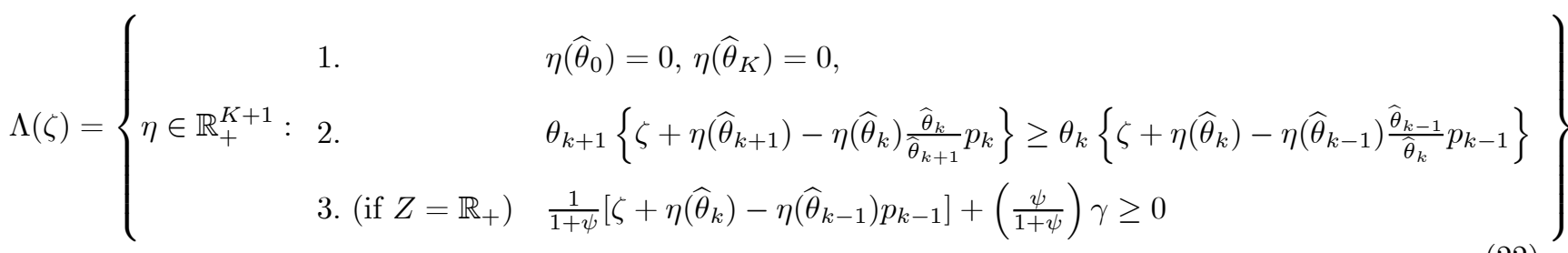

The second constraint in (22) implies that the weight on current utility is increasing in $k$, the third implies that the continuation Pareto-Negishi weight remains in $Z$ if $Z=\mathbb{R}_{+}$. Collectively, these conditions imply that $\Lambda$ is continuous, compact and convex-valued. The definition of our Bellman operator $T_{r}$ incorporates $\Lambda$ and is 
intuitive given (21):

$$
\begin{aligned}
T_{r} f(\zeta)=\inf _{\eta \in \Lambda(\zeta)} \sum_{\widehat{\theta}_{k} \in \Theta}\{W( & {\left.\left[\zeta+\eta\left(\widehat{\theta}_{k}\right)-\eta\left(\widehat{\theta}_{k-1}\right) \frac{\widehat{\theta}_{k-1}}{\widehat{\theta}_{k}} p_{k-1}\right] \widehat{\theta}_{k} ; q_{r}\right) } \\
& \left.+\lambda f\left(\frac{1}{1+\psi}\left[\zeta+\eta\left(\widehat{\theta}_{k}\right)-\eta\left(\widehat{\theta}_{k-1}\right) p_{k-1}\right]+\left(\frac{\psi}{1+\psi}\right) \gamma\right)\right\} \pi\left(\widehat{\theta}_{k}\right),
\end{aligned}
$$

With the definition of $T_{r}$ in place, we now formally establish that the value functions $\left\{V_{r}^{*}\right\}_{r=0}^{\infty}$ satisfy a sequence of Bellman equations.

Proposition 3 For all $r, V_{r}^{*}=T_{r} V_{r+1}^{*}$.

\section{Proof: See Appendix B.}

We complete this section by showing that the policy functions that solve the problems $V_{r}^{*}=T_{r} V_{r+1}^{*}$ induce an allocation that solves the component planner's problem (11). Let $\left\{\eta_{t}\right\}_{t=0}^{\infty}$ be a sequence of policy functions with each $\eta_{t}: Z \times\left(\left\{\widehat{\theta}_{0}\right\} \times \Theta\right) \rightarrow \mathbb{R}_{+}$. We say the utility allocation $\left\{u_{t}\right\}_{t=0}^{\infty}$ is induced by $\left\{\eta_{t}\right\}_{t=0}^{\infty}$ from $\zeta$ if $\zeta_{0}\left(\theta^{-1}\right)=\zeta$ and for each $\left(\theta^{t-1}, \widehat{\theta}_{k}\right)$,

$$
u_{t}\left(\theta^{t-1}, \widehat{\theta}_{k}\right)=\arg \sup _{u \in D} W\left(\left[\zeta_{t}\left(\theta^{t-1}\right)+\eta_{t}\left(\zeta_{t}\left(\theta^{t-1}\right), \widehat{\theta}_{k}\right)-\eta_{t}\left(\zeta_{t}\left(\theta^{t-1}\right), \widehat{\theta}_{k-1}\right) \frac{\widehat{\theta}_{k-1}}{\widehat{\theta}_{k}} p_{k-1}\right] \widehat{\theta}_{k} ; q_{r+t}\right)
$$

and

$$
\zeta_{t+1}\left(\theta^{t-1}, \widehat{\theta}_{k}\right)=\frac{1}{1+\psi}\left[\zeta_{t}\left(\theta^{t-1}\right)+\eta_{t}\left(\zeta_{t}\left(\theta^{t-1}\right), \widehat{\theta}_{k}\right)-\eta_{t}\left(\zeta_{t}\left(\theta^{t-1}\right), \widehat{\theta}_{k-1}\right) p_{k-1}\right]+\left(\frac{\psi}{1+\psi}\right) \gamma
$$

Lemma 2 Let $\left\{\eta_{r}^{*}\right\}_{r=0}^{\infty}$ denote a sequence of policy functions with $\eta_{r}^{*}: Z \times\left(\left\{\theta_{0}\right\} \cup \Theta\right) \rightarrow \mathbb{R}_{+}$such that for each $r$ and $\zeta, \eta_{r}^{*}\left(\zeta, \widehat{\theta}_{0}\right)=\eta_{r}^{*}\left(\zeta, \widehat{\theta}_{K}\right)=0$, while $\left\{\eta_{r}^{*}\left(\zeta, \widehat{\theta}_{k}\right)\right\}_{k=1}^{K-1}$ attains the infimum in

$$
V_{r}^{*}(\zeta)=\inf _{\eta \in \mathbb{R}_{+}^{K-1}} \sup _{\alpha \in \Omega_{1}} \mathcal{L}_{r}(\eta, \alpha ; \zeta)
$$

Then for each $r, 1) \eta_{r}^{*}$ is the unique policy function that attains the infima in $V_{r}^{*}=T_{r} V_{r+1}^{*}$;2) $\eta_{r}^{*}$ is continuous in $\zeta$, 3) if $\left\{u_{r+t}^{*}(\zeta)\right\}_{t=0}^{\infty}$ is a solution to (11) at $\zeta$ it is unique and is induced by $\left\{\eta_{r+t}^{*}\right\}_{t=0}^{\infty}$ from $\zeta$. 
Proof: See Appendix B.

The optimal multiplier policy functions $\left\{\eta_{r}^{*}\right\}_{r=0}^{\infty}$ may be used to construct a corresponding sequence of optimal Pareto-Negishi policy functions $\zeta^{*}=\left\{\zeta_{r+1}^{*}\right\}_{r=0}^{\infty}$ :

$$
\zeta_{r+1}^{*}\left(\zeta, \widehat{\theta}_{k+1}\right)=\frac{\psi}{1+\psi} \gamma+\frac{1}{1+\psi} \zeta+\frac{1}{1+\psi}\left\{\eta_{r}^{*}\left(\zeta, \widehat{\theta}_{k+1}\right)-\eta_{r}^{*}\left(\zeta, \widehat{\theta}_{k}\right) p_{k}\right\}
$$

These are central to our analysis and quite intuitive. As noted in the heuristic discussion, they incorporate a

shock term $\eta_{r}^{*}\left(\zeta, \widehat{\theta}_{k+1}\right)-\eta_{r}^{*}\left(\zeta, \widehat{\theta}_{k}\right) p_{k}$ with zero conditional expectation. Additionally, since $\lambda>\beta, \frac{1}{1+\psi} \in(0,1)$ and the $\left\{\zeta_{r+1}^{*}\right\}_{r=0}^{\infty}$ incorporate a force for mean reversion that draws the agent's Pareto-Negishi weight back towards $\gamma$.

\subsection{The component planner's dynamic programming problem}

So far we have shown that the optimal value functions $\left\{V_{r}^{*}\right\}_{r=0}^{\infty}$ solve the functional equations $V_{r}^{*}=T_{r} V_{r+1}^{*}$. However, we have not shown that they are the unique sequence of functions satisfying these equations, nor have we established a method for computing them. We address these important practical issues in this section. Specifically, we find sufficient conditions for $T_{r}$ to be a contraction. We then use the contractive property of the $\left\{T_{r}\right\}_{r=0}^{\infty}$ operators to establish a computational procedure for obtaining $\left\{V_{r}^{*}\right\}_{r=0}^{\infty}$. Since the functions $\left\{V_{r}^{*}\right\}_{r=0}^{\infty}$ are unbounded (in the sup norm), our arguments rely on finding a subset $\mathcal{V}$ of a weighted norm space such that each $V_{r}^{*} \in \mathcal{V}$ and $T_{r}: \mathcal{V} \rightarrow \mathcal{V}$. Before stating these arguments, we introduce some preliminary material on weighted norm spaces.

Let $w: Z \rightarrow[1, \infty)$ be a Borel-measurable function. For $f: Z \rightarrow \mathbb{R}$, define its $w$-norm by $\|f\|_{w}=$ $\sup _{\zeta \in Z} \frac{|f(\zeta)|}{w(\zeta)}$. We say that a function $f$ is $w$-bounded if $\|f\|_{w}<\infty$. Let $\mathbb{B}_{w}(Z)$ be the normed linear space of measurable, $w$-bounded functions with domain $Z . \mathbb{B}_{w}(Z)$ is a Banach space (see Hernández-Lerma and Laserre (1999)). 
Define the set of candidate value functions:

$$
\mathcal{V}=\{f: Z \rightarrow \mathbb{R}: f \text { is continuous and } \underline{V}(\zeta) \leq f(\zeta) \leq \bar{V}(\zeta)\}
$$

and suppose the existence of a weighting function satisfying the following assumption.

Assumption 3 There exists a weighting function $w: Z \rightarrow[1, \infty)$ such that 1) $\sup _{\zeta \in Z}\left|\frac{\underline{V}(\zeta)}{\overline{w(\zeta)}}\right|<\infty$ and $\sup _{\zeta \in Z}\left|\frac{\bar{V}(\zeta)}{w(\zeta)}\right|<\infty$, and 2) for some $\delta<1$ and all $\zeta \in Z$ and $\eta \in \Lambda(\zeta)$,

$$
\sup _{\zeta \in Z} \lambda \frac{\sum_{k=1}^{K} w\left(\frac{\zeta+\eta\left(\widehat{\theta}_{k}\right)-\eta\left(\widehat{\theta}_{k-1}\right) p_{k-1}+\psi}{1+\psi}\right)}{w(\zeta)} \in(0, \delta) .
$$

The first part of the assumption ensures that $\mathcal{V} \subset \mathbb{B}_{w}(Z)$. The second is a bound on the feasible growth of the Pareto-Negishi weights, we use it to show that $T_{r}$ satisfies a discounting property and is, hence, a contraction on $\mathcal{V}$. In Appendix $\mathrm{C}$, we find conditions on $u$ that are sufficient for a weight function satisfying Assumption 3 to exist. These conditions encompass many cases including CRRA utility with $\sigma>1$ and any bounded utility function.

Lemma 3 1) $T_{r}: \mathcal{V} \rightarrow \mathcal{V}$. 2) Under Assumption 3, $T_{r}$ is a contraction on $\mathcal{V}$

Proof: Appendix C.

Let $T_{r}^{s}=T_{r} \circ T_{r+1} \circ \cdots \circ T_{r+s}, s=0,1, \ldots$ Lemma 4 uses the contractive property of each $T_{r}$ to show that there is a unique sequence of functions in $\mathcal{V}$ satisfying the recursion $V_{r}^{\infty}=T_{r} V_{r+1}^{\infty}$ and that each element of this sequence can be obtained by iterating on the operators $\left\{T_{r}^{s}\right\}_{s=0}^{\infty}$.

Lemma 4 Let $V \in \mathcal{V}$ and let $V_{r}^{s}=T_{r}^{s} V$. Then 1) for each $r$, the sequence $\left\{V_{r}^{s}\right\}_{s=0}^{\infty}$ converges uniformly (in the w-norm) to a limiting function $V_{r}^{\infty} \in \mathcal{V}$; 2) for each $r, V_{r}^{\infty}=T_{r} V_{r}^{\infty}$; 3) $\left\{V_{r}^{\infty}\right\}_{r=0}^{\infty}$ is the unique sequence of functions in $\mathcal{V}$ satisfying the recursion for each $r, V_{r}^{\infty}=T_{r} V_{r+1}^{\infty}$. 
Proof: Appendix C.

Finally, we confirm that the functions $\left\{V_{r}^{\infty}\right\}$ equal the true value functions $\left\{V_{r}^{*}\right\}$.

Lemma 5 For all $r, V_{r}^{\infty}=V_{r}^{*}$.

Proof: Appendix C.

\section{The absence of immiseration and the existence of steady state}

\section{distributions}

\subsection{No immiseration}

We say that an agent is immiserated during shock history $\theta^{\infty}$ if its corresponding Pareto-Negishi weight sequence $\left\{\zeta_{t}\left(\theta^{t}\right)\right\}_{t=0}^{\infty}$ is absorbed by the non-positive real line, i.e. if $\lim \sup _{t \rightarrow \infty} \zeta_{t}\left(\theta^{t}\right) \leq 0$. It is readily verified that the continuation utility of an immiserated agent converges to its lowest bound. When $\beta=\lambda$, it is known that for a range of assumptions on $u$, agents are almost surely immiserated. As we have previously argued, this is an unappealing result from both a normative and a practical perspective. In stark contrast, when $\lambda>\beta$, it is immediate from the form of the optimal policy functions that immiseration cannot occur along any shock history. Observe that for $\zeta \leq 0$ and each $\widehat{\theta}_{k}, \eta_{t}^{*}\left(\zeta, \widehat{\theta}_{k}\right)=0$ and so

$$
\zeta_{t+1}^{*}\left(\zeta, \widehat{\theta}_{k}\right)=\frac{\psi}{1+\psi} \gamma+\frac{1}{1+\psi} \zeta \geq \zeta+\frac{\psi}{1+\psi} \gamma
$$

Consequently, if an agent's Pareto-Negishi weight enters the non-positive interval, it leaves with probability one. We have proved the following proposition.

Proposition 4 (No immiseration) For all histories $\theta^{\infty}, \lim _{\sup _{t \rightarrow \infty}} \zeta_{t+1}\left(\theta^{t}\right)>0$. 


\subsection{Stationary distributions in constant price economies}

When the sequence of resource prices are constant, $q_{t}=q$ for all $t$, we can obtain a sharper characterisation of the agent's Pareto-Negishi weight process $\left\{\zeta_{t+1}\right\}$. In this case, a specialisation of our earlier arguments can be used to show that the component planner's value and policy functions are time invariant. We denote them $V_{q}^{*}$, $\eta_{q}^{*}$ and $\zeta_{q}^{*}$ respectively. We have previously observed that the form of the optimal policy function $\zeta_{q}^{*}$ incorporates a force for mean reversion to $\gamma$. More formally, we now show that the Markov process for Pareto-Negishi weights induced by $\zeta_{q}^{*}$ satisfies a type of mixing. If the agent's Pareto-Negishi weight is less than $\gamma$ today, then it will almost surely be above $\gamma$ at some point in the future and vice versa. This sort of mixing can be interpreted as social mobility around the average Pareto-Negishi weight $\gamma \cdot{ }^{14}$ Realised sequences of low taste shocks result in the agent's Pareto-Negishi weight drifting above the average level $\gamma$. Since the agent's utility and consumption are functions of the Pareto weight, these rise to correspondingly higher levels. Sequences of high taste shocks take the agent's Pareto weight in the opposite direction.

Proposition 5 Let $\left\{\zeta_{t}\right\}$ denote the sequence of Pareto-Negishi weights induced by $\zeta_{q}^{*}$ from some $\zeta_{0}$. If $\zeta_{0}<\gamma$, then $\left\{\zeta_{t}\right\}$ reaches the set $\left\{\zeta_{t}>\gamma\right\}$ with probability one. If $\underline{\zeta}=\min \left\{0, \inf _{\mathbb{R}_{+} \times \Theta} \zeta_{q}^{*}\left(\zeta, \widehat{\theta}_{k}\right)\right\}>-\infty$, and $\zeta_{0}>\gamma$, then $\left\{\zeta_{t}\right\}$ reaches the set $\left\{\zeta_{t}<\gamma\right\}$ with probability one.

Proof: Suppose $\zeta_{0}<\gamma$. Let $\widetilde{\zeta}=\min \left\{\zeta_{0}, \min _{[0, \gamma] \times \Theta} \zeta_{q}^{*}\left(\zeta, \widehat{\theta}_{k}\right)\right\}$ and $\widehat{\zeta}=\max _{[0, \gamma] \times \Theta} \zeta_{q}^{*}\left(\zeta, \widehat{\theta}_{k}\right)$. Let $\left\{\zeta_{t}\right\}$ denote the process for Pareto-Negishi weights induced by $\zeta_{q}^{*}$ from $\zeta_{0}$. Define the random variable $T=\inf \left\{t: \zeta_{t}>\gamma\right\}$ and the stopped process $\zeta^{T}$ by $\zeta_{t}^{T}=\zeta_{\min \{t, T\}}$. Now, if $t \geq T, \zeta_{t+1}^{T}=\zeta_{t}^{T}$, otherwise, using the optimal policy function $\zeta_{q}^{*}, E_{t}\left[\zeta_{t+1}^{T}\right]=\frac{1}{1+\psi} \zeta_{t}+\left(\frac{\psi}{1+\psi}\right) \gamma \geq \zeta_{t}=\zeta_{t}^{T}$. Hence, $\left\{\zeta_{t}^{T}\right\}$ is a submartingale bounded on $[\widetilde{\zeta}, \widehat{\zeta}]$. Thus, $\left\{\zeta_{t}^{T}\right\}$ almost surely converges. By $(27)$, it cannot converge to a point on $[\widetilde{\zeta}, 0]$. Since the optimal multiplier functions $\eta_{q}^{*}\left(\zeta, \widehat{\theta}_{k}\right)$ are strictly positive on $(0, \infty)$ (see Appendix D), $\left\{\zeta_{t}^{T}\right\}$ cannot converge to a point on $(0, \gamma)$.

\footnotetext{
${ }^{14}$ It is easy to check that $\zeta_{q}^{*}$ implies $\lim _{t \rightarrow \infty} E\left[\zeta_{t}\right]=\gamma$ for any initial $\zeta_{0}$.
} 
We deduce that $T$ is almost surely finite and that the process $\left\{\zeta_{t}\right\}$ reaches the set $\left\{\zeta_{t}>\gamma\right\}$ with probability one.

Now assume that $\zeta_{0}>\gamma$. Note that for all $\left(\zeta, \widehat{\theta}_{k}\right) \in[\underline{\zeta}, \infty) \times \Theta, \zeta_{q}^{*}\left(\zeta, \theta_{k}\right) \in[\underline{\zeta}, \infty)$, so if $\underline{\zeta}>-\infty$, then the process $\left\{\xi_{t}\right\}$ with $\xi_{t}=\zeta_{t}-\underline{\zeta}$ takes its values in $[0, \infty)$. Define the stopping time $T=\inf \left\{t: \xi_{t}<\gamma-\underline{\zeta}\right\}$ and the stopped process $\xi^{T}=\left\{\xi_{t}^{T}\right\}_{t=0}^{\infty}$ by $\xi_{t}^{T}=\xi_{\min \{t, T\}}$. If $t \geq T, \xi_{t+1}^{T}=\xi_{t}^{T}$, otherwise, $E_{t}\left[\xi_{t+1}^{T}\right]=\frac{1}{1+\psi}\left(\xi_{t}-\underline{\zeta}\right)+$ $\left(\frac{\psi}{1+\psi}\right)(\gamma-\underline{\zeta}) \leq \xi_{t}-\underline{\zeta}=\xi_{t}^{T}$. Hence, $\xi^{T}$ is a non-negative supermartingale and it almost surely converges. By the same logic given above, it cannot converge to a point in $[\gamma-\underline{\zeta}, \infty)$. We deduce that $T$ is almost surely finite and that the process $\left\{\zeta_{t}\right\}$ reaches the set $\left\{\zeta_{t}<\gamma\right\}$ with probability one.

Proposition 5 assumes that the optimal policy function is bounded below on $\mathbb{R}_{+} \times \Theta$. Lemma B5 in the appendix establishes such boundedness for the case in which $u$ is bounded above (but not necessarily bounded below). Lemma B6, gives a condition for each $\zeta_{q}^{*}\left(\cdot, \widehat{\theta}_{k}\right)$ to be monotone. In this case $\inf _{\mathbb{R}_{+} \times \Theta} \zeta_{q}^{*}\left(\zeta, \widehat{\theta}_{k}\right) \geq$ $\zeta_{q}^{*}\left(0, \widehat{\theta}_{k}\right)=\frac{\psi}{1+\psi}$.

Let $P_{q}$ denote the Markov operator induced by $\zeta_{q}^{*}$ :

$$
P_{q} \Phi(H)=\int_{Z} \sum_{\widehat{\theta}_{k} \in \Theta} 1_{H}\left(\zeta_{q}^{*}\left(\zeta, \widehat{\theta}_{k}\right)\right) \pi\left(\widehat{\theta}_{k}\right) \Phi(d \zeta), \quad \Phi \in \mathcal{M}(Z), H \in \mathcal{B}(Z),
$$

where $\mathcal{M}(Z)$ is the space of probability measures on $Z, \mathcal{B}(Z)$ is the Borel sigma algebra on $Z$ and $1_{H}(\cdot)$ is the indicator function for the set $H$. Given an initial cross sectional distribution of Pareto-Negishi weights $\Phi_{0}$, repeated application of $P_{q}$ induces a sequence of cross sectional distributions $\left\{\Phi_{t}\right\}_{t=1}^{\infty}$ for subsequent periods. A basic question concerns the existence of an invariant probability measure for $P_{q}$. Such an invariant measure has an interpretation as a stationary cross sectional Pareto weight distribution for our environment. Since an agent's current consumption and utility is a function of its Pareto-Negishi weight and shock, it implies a stationary cross sectional distribution for these variables as well. We now show that such an invariant measure exists when $\zeta_{q}^{*}$ is bounded below on $\mathbb{R}_{+} \times \Theta$. 
Our proof of the existence of an invariant measure relies on an argument from the theory of iterated function systems, see Lasota and Mackay (1994). In order to state the result, we give a preliminary definition.

Definition 1 A stochastic system is a pair of sets $X$ and $Y$, a function $R: X \times Y \rightarrow X$ and a probability distribution $v$ on $Y$ satisfying the following. 1) $X$ is a closed subset of $\mathbb{R}^{d}$. $Y$ is a Borel subset of $\mathbb{R}^{k}$, 2) $R(\cdot, y)$ is continuous, each $y \in Y ; R(x, \cdot)$ is measurable, each $x \in X$, 3) the system evolves according to: $x_{t+1}=R\left(x_{t}, y_{t}\right)$, with each $y_{t}$ distributed iid according to $v$ and $x_{0}$ given.

A stochastic system induces a Markov operator $P$ according to $P \mu(H)=\int_{X} \int_{Y} 1_{H}(R(x, y)) v(d y) \mu(d x)$, where $\mu \in \mathcal{M}(X)$, and $H \in \mathcal{B}(X)$. The following result provides a sufficient condition for a stochastic system to admit an invariant measure.

Proposition 6 Let $P$ be the Markov operator corresponding to the stochastic system $(X, Y, R, v)$. Assume that there exists non-negative constants $\alpha_{0}$ and $\alpha_{1}, \alpha_{1}<1$ such that:

$$
\int_{Y}|R(x, y)| v(d y) \leq \alpha_{1}|x|+\alpha_{0} \quad \text { for all } x \in X .
$$

Then $P$ has an invariant distribution.

Proof: Lasota and Mackey (1994), Theorem 12.5.2, p. 420.

Remark 1: The analogue of the function $R$ in our analysis is the policy function $\zeta_{q}^{*}\left(\zeta, \widehat{\theta}_{k}\right)=\frac{\psi}{1+\psi} \gamma+\frac{1}{1+\psi} \zeta+$ $\eta_{q}^{*}\left(\zeta, \widehat{\theta}_{k}\right)-\eta_{q}^{*}\left(\zeta, \widehat{\theta}_{k-1}\right) p_{k-1}$ less a non-positive constant. Proposition 6 is useful because its key requirement is the moment condition (28). We know relatively little about $\zeta_{q}^{*}$ except that it is continuous and $\sum_{\widehat{\theta}_{k}} \zeta_{q}^{*}\left(\zeta, \widehat{\theta}_{k}\right) \pi\left(\widehat{\theta}_{k}\right)=$ $\frac{\psi}{1+\psi} \gamma+\frac{1}{1+\psi} \zeta$. We translate this fact into one concerning absolute values, as in (28), by a simple renormalisation. This is sufficient for an application of Proposition 6. Notice that Proposition 6 does not require the (often made) assumption of compactness of the state space which would clearly be restrictive in our setting. See Remark 2 below for further discussion. 
Our next proposition applies this result to our economy.

Proposition 7 Assume $\underline{\zeta}=\min \left\{\inf _{\mathbb{R}_{+} \times \Theta} \zeta_{q}^{*}\left(\zeta, \widehat{\theta}_{k}\right), 0\right\}>-\infty$. Then, $P_{q}$ admits an invariant distribution $\Phi_{q}$.

Proof: For all $\left(\zeta, \widehat{\theta}_{k}\right) \in[\underline{\zeta}, \infty) \times \Theta, \zeta_{q}^{*}\left(\zeta, \theta_{k}\right) \in[\underline{\zeta}, \infty)$. Since $\underline{\zeta}>-\infty, \xi_{q}^{*}(\xi, \theta):=\zeta_{q}^{*}(\xi+\underline{\zeta}, \theta)-\underline{\zeta}$ maps $[0, \infty)$ into itself. Set $X=[0, \infty), Y=\Theta, v=\pi$, and $R=\xi_{q}^{*}$. Note that for $\xi \geq 0$

$$
\begin{aligned}
\sum_{k}\left|\xi_{q}^{*}\left(\xi, \widehat{\theta}_{k}\right)\right| \pi\left(\widehat{\theta}_{k}\right) & =\sum_{k} \xi_{q}^{*}\left(\xi, \widehat{\theta}_{k}\right) \pi\left(\widehat{\theta}_{k}\right)=\sum_{k}\left\{\zeta_{q}^{*}\left(\xi+\underline{\zeta}, \widehat{\theta}_{k}\right)-\underline{\zeta}\right\} \pi\left(\widehat{\theta}_{k}\right) \\
& =\sum_{k}\left\{\frac{\xi+\underline{\zeta}+\psi+\eta_{q}^{*}\left(\xi+\underline{\zeta}, \widehat{\theta}_{k+1}\right)-\eta_{q}^{*}\left(\xi+\underline{\zeta}, \widehat{\theta}_{k}\right) p_{k}}{1+\psi}-\underline{\zeta}\right\} \pi\left(\widehat{\theta}_{k}\right) \\
& =\frac{\xi}{1+\psi}+\frac{\psi}{1+\psi}\{1-\underline{\zeta}\} .
\end{aligned}
$$

Where the first equality uses $\xi_{q}^{*}\left(\xi, \widehat{\theta}_{k}\right) \geq 0$, the second and third the definitions of $\xi_{q}^{*}$ and $\zeta_{q}^{*}$ and the fourth $\sum_{k}\left\{\eta_{q}^{*}\left(\xi+\underline{\zeta}, \widehat{\theta}_{k+1}\right)-\eta_{q}^{*}\left(\xi+\underline{\zeta}, \widehat{\theta}_{k}\right) p_{k}\right\} \pi\left(\widehat{\theta}_{k}\right)=0$. Setting $\alpha_{1}=\frac{1}{1+\psi} \in(0,1)$ and $\alpha_{0}=\frac{\psi}{1+\psi}\{1-\underline{\zeta}\} \geq 0$ verifies (28). $R\left(\cdot, \widehat{\theta}_{k}\right)=\xi_{q}^{*}\left(\cdot, \widehat{\theta}_{k}\right)$ is continuous since $\zeta_{q}^{*}\left(\cdot, \widehat{\theta}_{k}\right)$ is. It then follows from Proposition 6 that the process for $\xi$ admits an invariant distribution. Consequently so too does the process for $\zeta$.

It is easy to see that $\Phi_{q}$ is non-degenerate. In particular, it follows from our discussion of immiseration that $\Phi_{q}(0, \infty)>0$. This is in contrast to the equal discounting case $\lambda=\beta$, when the immiseration result implies that $\Phi_{q}$ puts all mass on the non-positive Pareto-Negishi weights. We now show that when $\zeta_{q}^{*}$ is monotone in $\zeta, \Phi_{q}$ is unique and any sequence of measures induced by $P_{q}$ converges strongly and at a geometric rate to $\Phi_{q}$. This ergodic result strengthens our earlier conclusions about mixing and social mobility. Following Phelan (2003), multiple ergodic sets can be interpreted as caste systems: sets of Pareto-Negishi weights from which an agent cannot escape. Our results imply a unique ergodic set and, hence, the absence of (multiple) caste systems.

We prove our ergodicity result in two steps. First, we establish that $P_{q}$ has a unique invariant probability measure and that any sequence of probability measures induced by $P_{q}$ from some arbitrary $\Phi_{0} \in \mathcal{M}(Z)$ converges 
weakly to this invariant measure. We follow Lasota and Mackey in referring to Markov operators with these properties as weakly asymptotic. We then build on this to establish the geometric ergodicity result. Our first step uses the following theorem of Lasota and Mackey.

Proposition 8 Let $P$ be the Markov operator corresponding to $(X, Y, R, v)$. Assume that:

$$
\int_{Y}(|R(x, y)-R(z, y)|) v(d y) \leq \alpha_{1}|x-z|, \quad x, z \in X
$$

and $\int_{Y}(|R(0, y)|) v(d y) \leq \alpha_{0}$, where $\alpha_{1} \in(0,1)$ and $\alpha_{0} \in(0, \infty)$. Then the system $(X, Y, R, v)$ is weakly asymptotically stable.

Proof: Lasota and Mackey (1994), Theorem 12.6.1, p. 423.

The next proposition applies this result to the stochastic system implied by $\left(Z, \Theta, \zeta_{q}^{*}, \pi\right)$.

Proposition 9 Assume that each $\zeta_{q}^{*}\left(\cdot, \widehat{\theta}_{k}\right)$ is monotone, then $P_{q}$ is weakly asymptotically stable.

Proof: Set $X=Z, Y=\Theta, R=\zeta_{q}^{*}$ and $v=\pi$. Suppose $\zeta \geq \zeta^{\prime}$, then

$$
\begin{aligned}
\sum_{k}\left|\zeta_{q}^{*}\left(\zeta, \widehat{\theta}_{k}\right)-\zeta_{q}^{*}\left(\zeta^{\prime}, \widehat{\theta}_{k}\right)\right| \pi(\theta) & =\sum_{k}\left(\zeta_{q}^{*}\left(\zeta, \widehat{\theta}_{k}\right)-\zeta_{q}^{*}\left(\zeta^{\prime}, \widehat{\theta}_{k}\right)\right) \pi\left(\widehat{\theta}_{k}\right) \\
& =\sum_{k}\left(\frac{\zeta+\psi+\eta_{q}^{*}\left(\zeta, \widehat{\theta}_{k+1}\right)-\eta_{q}^{*}\left(\zeta, \widehat{\theta}_{k}\right) p_{k}}{1+\psi}-\frac{\zeta^{\prime}+\psi+\eta_{q}^{*}\left(\zeta^{\prime}, \widehat{\theta}_{k+1}\right)-\eta_{q}^{*}\left(\zeta^{\prime}, \widehat{\theta}_{k}\right) p_{k}}{1+\psi}\right) \pi\left(\widehat{\theta}_{k}\right) \\
& =\frac{\zeta-\zeta^{\prime}}{1+\psi}=\frac{\left|\zeta-\zeta^{\prime}\right|}{1+\psi}
\end{aligned}
$$

where we use the monotonicity of each $\zeta_{q}^{*}\left(\cdot, \widehat{\theta}_{k}\right)$ in the first line, the definition of $\zeta_{q}^{*}$ in the second, and $\sum_{k}\left\{\eta_{q}^{*}\left(\widehat{\zeta}, \widehat{\theta}_{k+1}\right)-\eta_{q}^{*}\left(\widehat{\zeta}, \widehat{\theta}_{k}\right) p_{k}\right\} \pi\left(\widehat{\theta}_{k}\right)=0$ at $\widehat{\zeta}=\zeta$ and $\zeta^{\prime}$ in the third. The argument for $\zeta \leq \zeta^{\prime}$ is very similar. This verifies (29) with $\alpha_{1}=\frac{1}{1+\psi}$. To verify the second condition in Proposition 8 , use the definition of $\zeta_{q}^{*}$ and, for all $k, \eta_{q}^{*}\left(0, \widehat{\theta}_{k}\right)=0$, to obtain: $\sum_{k}\left|\zeta_{q}^{*}\left(0, \widehat{\theta}_{k}\right)\right| \pi\left(\widehat{\theta}_{k}\right)=\sum_{k}\left|\frac{\psi+\eta_{q}^{*}\left(0, \widehat{\theta}_{k+1}\right)-\eta_{q}^{*}\left(0, \widehat{\theta}_{k}\right) p_{k}}{1+\psi}\right| \pi\left(\widehat{\theta}_{k}\right)=\frac{\psi}{1+\psi}$. The result then follows from Proposition 8. 
Remark 2: We give conditions for $\zeta_{q}^{*}$ to be monotone in Appendix B. These require that the shock spread not be too large and that $\partial W / \partial \rho$ be concave, where $W(\rho ; q)=\sup _{u \in D} \rho u-q C(u)$. The latter is satisfied if $u$ is CRRA with $\sigma>1 / 2$. There is a long tradition of studying Markov processes induced by monotone policy functions in economics, see for example Hopenhayn and Prescott (HP) (1992). For the most part these results rely on a compact state space. In the context of the present model, this is restrictive. Proposition 9 does not require such a condition. Additionally, to obtain uniqueness and weak asymptotic stability, most contributions to the economics literature supplement monotonicity with a mixing condition, see HP, Theorem 2. In the present model, the underlying mean reversion property of the $\zeta_{q}^{*}$, coupled with monotonicity, ensures the contractivity condition (29) and no additional mixing conditions are needed.

To strengthen Proposition 9 and obtain the geometric ergodicity result, it is necessary to embed $\mathcal{M}(Z)$ into a normed space. Let $w: Z \rightarrow[1, \infty)$ be a weight function. If $\mu$ is a signed measure on $\mathcal{B}(Z)$ with total variation $|\mu|$, denote its $w$-norm by $\|\mu\|_{w}=\int_{Z} w d|\mu|$. Let $\mathbb{M}_{w}(Z)$ denote the set of signed measures on $\mathcal{B}(Z)$ with finite $w$-norm. $P: Z \times \mathcal{B}(Z) \rightarrow[0,1]$ is a signed kernel if for each $\zeta \in Z, P(\zeta, \cdot)$ is a signed measure on $Z$ and for all $H \in \mathcal{B}(Z), P(\cdot, H)$ is a measurable function. If $P$ is a signed kernel define its $w$-norm by: $\|P\|_{w}=$ $\sup _{\mu \in\left\{\mathbb{M}_{w}(Z):\|\mu\|_{w} \leq 1\right\}}\|P \mu\|_{w}$.

Definition 2 Let $w: Z \rightarrow[1, \infty)$ be a weight function and $P$ a Markov operator such that $\|P\|_{w}<\infty$. Then $P$ is called w-geometrically ergodic with convergence rate $\rho \in(0,1)$ if there is a $\mu_{*} \in \mathbb{M}_{w}(Z)$ and $R \geq 0$ such that for all $t\left\|P^{t}-\mu_{*}\right\|_{w} \leq R \rho^{t}$.

Thus, if a Markov operator $P$ is $w$-geometrically ergodic, it has a unique invariant measure and any sequence of measures induced by $P$ converges strongly and at a geometric rate $\rho$ to this measure.

Proposition 10 Define $w: Z \rightarrow[1, \infty)$ pointwise by $w(\zeta)=1+|\zeta|$. Assume that each $\zeta_{q}^{*}\left(\cdot, \widehat{\theta}_{k}\right)$ is monotone. Then $P_{q}$ is w-geometrically ergodic with convergence rate $\frac{1}{1+\psi}$. 
Proof: By the previous proposition $P_{q}$ is weakly asymptotically stable. Hence, it has a unique invariant measure, $\mu_{q}$. It follows that the Markov process associated with $P_{q}$ is $\mu_{q}$-irreducible and aperiodic. By Theorem 7.3.10 p. 12, Hernández-Lerma and Laserre, $P_{q}$ is $w$-geometrically ergodic with convergence rate $\rho \in(0,1)$ if, in addition, for all $\zeta$ and some $b \in \mathbb{R}, \sum_{k} w\left(\zeta_{q}^{*}\left(\zeta, \widehat{\theta}_{k}\right)\right) \pi\left(\widehat{\theta}_{k}\right) \leq \rho w(\zeta)+b$. To complete the proof, we verify this condition.

Now,

$$
\begin{aligned}
\sum_{k}\left(1+\left|\zeta_{q}^{*}\left(\zeta, \widehat{\theta}_{k}\right)\right|\right) \pi\left(\widehat{\theta}_{k}\right) & =\sum_{k}\left(1+\left|\frac{\zeta+\psi+\eta_{q}^{*}\left(\zeta, \widehat{\theta}_{k+1}\right)-\eta_{q}^{*}\left(\zeta, \widehat{\theta}_{k}\right) p_{k}}{1+\psi}\right|\right) \pi\left(\widehat{\theta}_{k}\right) \\
& \leq \frac{1}{1+\psi}(1+|\zeta|)+\frac{2 \psi}{1+\psi}
\end{aligned}
$$

The first line above uses the definition of $\zeta_{q}^{*}$. The second line uses the fact that if $\zeta \leq 0$, for all $k, \eta_{q}^{*}\left(\zeta, \widehat{\theta}_{k}\right)=0$, while if $\zeta \geq 0$, by monotonicity, $\zeta_{q}^{*}\left(\zeta, \widehat{\theta}_{k}\right) \geq \zeta_{q}^{*}\left(0, \widehat{\theta}_{k}\right)=\frac{\psi}{1+\psi}>0$ and $\sum_{k}\left\{\eta_{q}^{*}\left(\zeta, \widehat{\theta}_{k+1}\right)-\eta_{q}^{*}\left(\zeta, \widehat{\theta}_{k}\right) p_{k}\right\} \pi\left(\widehat{\theta}_{k}\right)=0$. This confirms the condition with $\rho=\frac{1}{1+\psi}$ and $b=\frac{2 \psi}{1+\psi}$, proving the result.

\section{The Atkeson-Lucas unemployment insurance problem}

The analysis developed in this paper can be applied to variety of other environments. One such is the unemployment insurance model of Atkeson and Lucas (1995). We sketch this application here. An agent receives either a positive or a zero productivity shock in each period. The former is interpreted as a job, the latter as unemployment. Thus, we set $\Theta=\{E, U\}$, for employment and unemployment respectively. These shocks are private information. If the agent has a job, she is able to exert effort in work. Here preferences over consumption and effort are given by:

$$
\sum_{t=0}^{\infty} \beta^{t}\left[u\left(c_{t}\left(\theta^{t}\right)\right)-1_{E}\left(\theta_{t}\right) v\left(y_{t}\left(\theta^{t}\right)\right)\right] \pi\left(\theta^{t}\right)
$$


where $u: \mathbb{R}_{+} \rightarrow D$ is as before, $v: L \rightarrow B$ is convex, increasing and differentiable and $1_{E}$ is an indicator function for the employment state. Let $Y=v^{-1}$. Atkeson and Lucas assume that $L=[0, \bar{y}]$ and that $v$ is linear. To avoid considering corner solutions we will assume that $v$ is strictly convex, $\lim _{y \downarrow 0} v^{\prime}(y)=0$, and $\lim _{y \uparrow \bar{y}} v^{\prime}(y)=\infty$. We will also assume that $u$ is bounded above.

The planner's problem proceeds as in the previous environment, with appropriate respecifications of shocks and preferences. Assuming constant prices, the recursive component planner problem is:

$$
V(\zeta)=\left[W^{E}(\zeta+\eta, q)+\lambda V\left(\frac{\zeta+\eta+\psi}{1+\psi}\right)\right] \pi(E)+\left[W^{U}(\zeta-\eta p, q)+\lambda V\left(\frac{\zeta-\eta p+\psi}{1+\psi}\right)\right] \pi(U),
$$

where $W^{E}(\rho, q):=\sup _{u \in D, v \in B} \rho\{u-v\}-q\{C(u)-Y(v)\}$ and $W^{U}(\rho, q):=\sup _{u \in D} \rho u-q C(u) . \operatorname{Let}_{q}^{*}(\zeta, \theta)$ denote the optimal continuation Pareto-Negishi weight function, and $\eta^{*}(\zeta)$ the optimal Lagrange multiplier policy function. A convenient feature of this problem is that both $\zeta_{q}^{*}(\zeta, E)$ and $\zeta_{q}^{*}(\zeta, U)$ are increasing, under no additional assumptions. Thus, the implied Markov operator $P_{q}$ is $w$-geometric with unique invariant measure $\mu_{q}^{*}$. Since $\eta_{q}^{*}(0)=0$ as in our earlier environment, we have that $\zeta_{q}^{*}(\zeta, E) \geq \zeta_{q}^{*}(\zeta, U) \geq \zeta_{q}^{*}(0, U)=\frac{\psi}{1+\psi}$. Thus, an agent's utility from consumption is bounded below by $u=\arg \sup _{u \in D} \frac{\psi}{1+\psi} u-q C(u)$, her distutility from effort is bounded above by: $v=\arg \sup _{v \in B}-\frac{\psi}{1+\psi} v+q Y(v)$.

\section{Social credibility and social discounting}

The immiseration result, obtained when the planner and the agent discount at the same rate, requires a social commitment to treat almost everyone arbitrarily badly in the long run. This degree of social commitment seems implausible, and provides a motivation for analysing environments that incorporate restrictions on the ability of the planner or, more generally, society to commit. In this section, we describe a class of such commitment or credibility-constrained environments and develop a connection between them and the high planner discount economies of previous sections. 
Consider then an economy that both specialises and augments that described in Section 2. It specialises the earlier setting by assuming a common discount factor $\beta$ for the planner and the agent, and a time invariant Pareto-Negishi weight $\zeta_{0}$ for all agents. It augments this setting with a sequence of credibility constraints. These require that the planner maintain a weighted aggregate of agent continuation utilities above a lower bound. This weighted aggregate may or may not be the same as the weighted aggregate in the planner's own objective. Although the planner might propose an allocation that violates these constraints, in an attempt to reduce the social cost (from her perspective) of truthful revelation, the allocation will not be viable and agents will not find it credible. We assume that the credibility constraints stem from the ability of agents to collectively resist or otherwise avoid reductions in their continuation utility, even if they, or their ancestors, previously accepted an allocation that called for such reductions. This resistance might take the form of social disruption, political pressure, or a straightforward lack of commitment on the part of a benevolent planner. Our model encompasses all of these cases. Consequently, we will think of it as a reduced form for a family of political economy-type games in which the credibility constraints are endogenously derived as equilibrium conditions. Before proceeding, we give a specific example taken from Sleet and Yeltekin (2004).

Example (Sleet and Yeltekin (2004)). Suppose that the planner and the agents share a common discount factor and the planner cannot commit to implementing an allocation. Agents are indexed by a single time invariant Pareto weight $\zeta_{0}$. Consider the following game. At the beginning of each period $t$, the planner selects an allocation function $\left\{u_{t}^{\zeta_{0}}\right\}_{\zeta_{0} \in Z}$, with $u_{t}^{\zeta_{0}}: \Theta^{t+1} \rightarrow D$. Next agents observe their current shock $\theta_{t}$ and make a report $\delta_{t} \in \Theta$ concerning its value. The planner then awards current utility to agents contingent on their Pareto weight and their history of shock reports according to $\left\{u_{t}^{\zeta_{0}}\right\}_{\zeta_{0} \in Z}$. At no point in time is the planner committed to implementing any continuation allocation. Let $\mathcal{U}_{t}$ denote the set of $t$-period allocation functions and let $\mathcal{U}^{t}:=\prod_{s=0}^{t-1} \mathcal{U}_{s}$, with $\mathcal{U}^{0}=\emptyset$. We can then define a planner strategy to be a collection of functions 
$\sigma=\left\{\sigma_{t}\right\}_{t=0}^{\infty}$ with $\sigma_{t}: \mathcal{U}^{t} \rightarrow \mathcal{U}_{t}$. Each $\sigma_{t}$ maps from an "aggregate history" of allocation functions previously chosen by the planner to a current allocation function. The period $t$ individual history of an agent $\left(u^{t+1}, \zeta_{0}, \theta^{t}\right) \in$ $\mathcal{U}^{t+1} \times Z \times \Theta^{t+1}$ augments the aggregate history $u^{t+1}$ with the agent's Pareto weight and history of taste shocks. A reporting policy is now a sequence of functions $\delta=\left\{\delta_{t}\right\}_{t=0}^{\infty}$, with each $\delta_{t}$ defined on the larger set of individual histories: $\delta_{t}: \mathcal{U}^{t+1} \times Z \times \Theta^{t+1} \rightarrow \Theta$.

Using this notation, we may define a credible equilibrium to be a pair $(\sigma, \delta)$ such that 1$)$ after all individual histories, it is optimal for agents to use the reporting strategy $\delta$ and 2) after all aggregate histories, it is optimal for the planner to use strategy $\sigma$. In Sleet and Yeltekin (2004), we show that the worst credible equilibrium payoff is the autarkic one, $U_{\text {aut }}$, i.e. the one that is obtained from an allocation that delivers no insurance to an agent at each date. Using an argument similar to Abreu (1988), we then show that an allocation is credible (i.e. is supported by a credible equilibrium) only if the planner is better off adhering to the allocation rather than deviating and triggering a reversion to autarky. Consequently, the optimal credible allocation solves:

$$
\begin{aligned}
\sup _{\left\{\alpha^{\zeta_{0}} \in A\right\}} \int_{Z} \zeta_{0} \sum_{t=0}^{\infty} \beta^{t} \sum_{\theta^{t} \in \Theta^{t+1}} \theta_{t} u_{t}^{\zeta_{0}}\left(\theta^{t}\right) \pi^{t}\left(\theta^{t}\right) \Phi_{0}\left(d \zeta_{0}\right) \\
-\int_{Z} \sum_{t=0}^{\infty} \widehat{q}_{t} \sum_{\theta^{t} \in \Theta^{t+1}} C\left(u_{t}^{\zeta_{0}}\left(\theta^{t}\right)\right) \pi^{t}\left(\theta^{t}\right) \Phi_{0}\left(d \zeta_{0}\right)
\end{aligned}
$$

subject to for $\Phi_{0}$-a.e. $\zeta_{0}$, for all $t, \theta^{t-1}, k<K, \Delta U_{t}\left(\alpha^{\zeta_{0}}\left(\theta^{t-1}\right), \widehat{\theta}_{k}, \widehat{\theta}_{k+1}\right) \geq 0$ and for all $t$,

$$
\int_{Z} \zeta_{0} \sum_{\theta^{t-1} \in \Theta^{t}} U_{t}\left(\alpha^{\zeta_{0}}\left(\theta^{t-1}\right)\right) \pi^{t-1}\left(\theta^{t-1}\right) \Phi_{0}\left(d \zeta_{0}\right) \geq U_{\text {aut }}
$$

Constraints (31) are the credibility constraints for this problem. In this example, the lower bound is set to the autarkic payoff, while the weighting in the credibility constraints coincides with that in the planner's objective.

In the remainder of this section, we write down and formulate a Lagrangian for a general credibilityconstrained problem. We then construct a new endogenous discounting scheme from the Lagrange multipliers on 
the credibility constraints. This construction connects the credibility-constrained and the high planner discount environments. The value of such a connection is two fold. First, it provides a rationale for the analysis of high planner discount economies. Second, results from the high planner discount economy can be applied to the credibility-constrained economy. In particular, we will be able to use results on the existence of a stationary solution to the component planner problem with constant resource prices, to establish the existence of "stationary credible equilibria": optimal allocations that satisfy a credibility constraint and imply a steady state distribution over Pareto-Negishi weights.

More formally suppose that the planner solves the following credible social insurance problem:

$$
\begin{aligned}
\sup _{\left\{\alpha\left(\zeta_{0}, \gamma\right) \in A\right\}} \int_{Z} \int_{Z} \zeta_{0} \sum_{t=0}^{\infty} \beta^{t} \sum_{\theta^{t} \in \Theta^{t+1}} \theta_{t} u_{t}^{\left(\zeta_{0}, \gamma\right)}\left(\theta^{t}\right) \pi^{t}\left(\theta^{t}\right) \Phi_{0}\left(d \zeta_{0}\right) \Psi_{0}(d \gamma) \\
-\int_{Z} \int_{Z} \sum_{t=0}^{\infty} \widehat{q}_{t} \sum_{\theta^{t} \in \Theta^{t+1}} C\left(u_{t}^{\left(\zeta_{0}, \gamma\right)}\left(\theta^{t}\right)\right) \pi^{t}\left(\theta^{t}\right) \Phi_{0}\left(d \zeta_{0}\right) \Psi_{0}(d \gamma)
\end{aligned}
$$

subject to for $\Phi_{0}$-a.e. $\zeta_{0}$, for $\Psi_{0}$-a.e. $\gamma$, for all $t, \theta^{t-1}, k<K, \Delta U_{t}\left(\alpha^{\left(\zeta_{0}, \gamma\right)}\left(\theta^{t-1}\right), \widehat{\theta}_{k}, \widehat{\theta}_{k+1}\right) \geq 0$ and for all $t$,

$$
\int_{Z} \int_{Z} \gamma \sum_{\theta^{t-1} \in \Theta^{t}} U_{t}\left(\alpha^{\left(\zeta_{0}, \gamma\right)}\left(\theta^{t-1}\right)\right) \pi^{t-1}\left(\theta^{t-1}\right) \Phi_{0}\left(d \zeta_{0}\right) \Psi(d \gamma) \geq \underline{U}
$$

The constraints (33) require that the planner maintains a $\gamma$-weighted aggregate of agent continuation utilities above a lower bound. Consistent with the discussion above, we interpret the $\gamma$-weight as capturing the relative ability of an agent (or dynasty) to resist or avoid reductions in its continuation utility. Notice that since (33) is applied to the entire population of agents, the planner is free to immiserate any individual agent. However, it can no longer treat the entire population of agents arbitrarily badly. In contrast to our earlier example, we allow the $\gamma$-weights to differ from those in the planner's objective. ${ }^{15}$ We also treat the lower bound $\underline{U}$ as a parameter (in the example above, it is given by the autarkic utility). We call (33) a social credibility constraint and refer to (32) as the credible social insurance problem at $\left\{\left\{\widehat{q}_{t}\right\}_{t=0}^{\infty}, \underline{U}, \Phi_{0}, \Psi_{0}\right\}$.

\footnotetext{
${ }^{15}$ We do assume that both $\zeta_{0}$ and $\gamma$ are constants. More generally still, they could be time varying or stochastic.
} 
Denoting the multiplier on the $t$-th social credibility constraint (33) by $\beta^{t} \psi_{t} \prod_{s=0}^{t-1}\left(1+\psi_{t}\right)$, we construct the following Lagrangian for problem (32):

$$
\begin{aligned}
& \sup _{\left\{\alpha_{\left(\zeta_{0}, \gamma\right)} \in A\right\}} \int_{Z} \int_{Z} \zeta_{0} U_{0}\left(\alpha^{\left(\zeta_{0}, \gamma\right)}\right) \Phi_{0}\left(d \zeta_{0}\right) \Psi_{0}(d \gamma)-\sum_{t=0}^{\infty} \widehat{q}_{t} \int_{Z} \int_{Z} \sum_{\theta^{t} \in \Theta^{t+1}} C\left(u_{t}^{\left(\zeta_{0}, \gamma\right)}\left(\theta^{t}\right)\right) \pi^{t}\left(\theta^{t}\right) \Phi_{0}\left(d \zeta_{0}\right) \Psi_{0}(d \gamma) \\
& \quad+\sum_{t=0}^{\infty} \beta^{t} \psi_{t} \prod_{s=0}^{t-1}\left(1+\psi_{t}\right)\left[\int_{Z} \int_{Z} \gamma \sum_{\theta^{t-1} \in \Theta^{t}} U_{t}\left(\alpha^{\left(\zeta_{0}, \gamma\right)}\left(\theta^{t-1}\right)\right) \pi^{t-1}\left(\theta^{t-1}\right) \Phi_{0}\left(d \zeta_{0}\right) \Psi_{0}(d \gamma)-\underline{U}\right]
\end{aligned}
$$

subject to for $\Phi_{0}$-a.e. $\zeta_{0}$, for $\Psi_{0}$-a.e. $\gamma$, for all $t, \theta^{t-1}, k<K, \Delta U_{t}\left(\alpha^{\left(\zeta_{0}, \gamma\right)}\left(\theta^{t-1}\right), \widehat{\theta}_{k}, \widehat{\theta}_{k+1}\right) \geq 0$. Notice that this Lagrangian absorbs the social credibility (but not the incentive) constraints into the objective. If the utility functions of the agents are bounded either above or below and if $\sum_{t=0}^{\infty} \beta^{t} \psi_{t} \prod_{s=0}^{t-1}\left(1+\psi_{t}\right)<\infty$, the Lagrangian (34) may be rearranged to give the following optimisation:

$$
\begin{aligned}
\sup _{\left\{\alpha\left(\zeta_{0}, \gamma\right) \in A\right\}} \int_{Z} & \int_{Z} \sum_{t=0}^{\infty} B_{0}^{t} \gamma_{t}\left(\zeta_{0}, \gamma\right) \sum_{\theta^{t} \in \Theta^{t+1}} \theta_{t} u_{t}^{\left(\zeta_{0}, \gamma\right)}\left(\theta^{t}\right) \pi^{t}\left(\theta^{t}\right) \Phi_{0}\left(d \zeta_{0}\right) \Psi_{0}(d \gamma) \\
& -\sum_{t=0}^{\infty} q_{t} B_{0}^{t} \int_{Z} \int_{Z} \sum_{\theta^{t} \in \Theta^{t+1}} C\left(u_{t}^{\left(\zeta_{0}, \gamma\right)}\left(\theta^{t}\right)\right) \pi^{t}\left(\theta^{t}\right) \Phi_{0}\left(d \zeta_{0}\right) \Psi_{0}(d \gamma),
\end{aligned}
$$

subject to for $\Phi_{0}$-a.e. $\zeta_{0}$, for $\Psi_{0}$-a.e. $\gamma$, for all $t, \theta^{t-1}, k<K, \Delta U_{t}\left(\alpha^{\left(\zeta_{0}, \gamma\right)}\left(\theta^{t-1}\right), \widehat{\theta}_{k}, \widehat{\theta}_{k+1}\right) \geq 0$. Here $q_{t}=\widehat{q}_{t} / B_{0}^{t}$, $B_{0}^{t}=\beta^{t} \prod_{s=0}^{t-1}\left(1+\psi_{s}\right)$ and $\gamma_{t}\left(\zeta_{0}, \gamma\right)=\gamma+\psi_{t}+\frac{\left(\zeta_{0}-\gamma\right)}{\prod_{s=0}^{t-1}\left(1+\psi_{s}\right)}$. It then follows directly from (35), that the planner's credible social insurance problem (32) can be transformed into one without credibility constraints, but with a new (time varying) discounting scheme $\left\{B_{0}^{t}\right\}_{t=0}^{\infty}$ and a new Pareto weighting scheme $\left\{\gamma_{t}\right\}_{t=0}^{\infty}$. Since for all $t, B_{0}^{t} \geq \beta^{t}$, a credibility-constrained planner will behave as if she has a higher discount factor and is more patient than the agents. In this sense credibility is a force for social patience. Notice also that the formula for $\gamma_{t}\left(\zeta_{0}, \gamma\right)$ implies that it is an agent's weight in the credibility constraint $\gamma$ that determines her long run Pareto-Negishi weight in the transformed problem. Hence, it will be $\gamma$, not the weight in the planner's preferences $\zeta_{0}$, that determines the point to which an agent's endogenous Pareto-Negishi weight mean reverts in the recursive formulation of the problem below. ${ }^{16}$ More formally, the following result links credible social insurance problems to problems with

\footnotetext{
${ }^{16}$ There is one small difference between the high planner discount economy and the economy with credibility constraints. In the
} 
relatively patient planners. The proof is obtained by a modification of an argument in Sleet and Yeltekin (2004) and is omitted.

Proposition 11 Fix $\left\{\left\{\widehat{q}_{t}\right\}_{t=0}^{\infty}, \underline{U}, \Phi_{0}, \Psi_{0}\right\}$ with $\left\{\widehat{q}_{t}\right\}_{t=0}^{\infty} \in \ell_{+}^{1}$ and suppose there exists an allocation $\left\{\alpha^{\left(\zeta_{0}, \gamma\right)}\right\}$ and a sequence $\left\{\psi_{t}\right\} \in \mathbb{R}_{+}^{\infty}$ that satisfy the following conditions:

1. $\sum_{t=0}^{\infty} \beta^{t} \psi_{t} \prod_{s=0}^{t-1}\left(1+\psi_{s}\right)<\infty$;

2. $\psi_{t}\left(\int_{Z} \int_{Z} \sum_{\theta^{t} \in \Theta^{t+1}} \gamma U_{t}\left(\alpha^{\left(\zeta_{0}, \gamma\right)}\left(\theta^{t}\right)\right) \pi^{t}\left(\theta^{t}\right) \Phi_{0}\left(d \zeta_{0}\right) \Psi_{0}(d \gamma)-\underline{U}\right)=0$

and $\int_{Z} \int_{Z} \sum_{\theta^{t} \in \Theta^{t+1}} \gamma U_{t}\left(\alpha^{\left(\zeta_{0}, \gamma\right)}\left(\theta^{t}\right)\right) \pi^{t}\left(\theta^{t}\right) \Phi_{0}\left(d \zeta_{0}\right) \Psi_{0}(d \gamma)-\underline{U} \geq 0$

3. each $\alpha^{\left(\zeta_{0}, \gamma\right)}$ solves $\sup _{\left\{u_{t}\right\}_{t=0}^{\infty} \in A} \sum_{t=0}^{\infty} \sum_{\theta^{t} \in \Theta^{t+1}} B_{0}^{t}\left[\gamma_{t}\left(\zeta_{0}, \gamma\right) \theta_{t} u_{t}\left(\theta^{t}\right)-q_{t} C\left(u_{t}\left(\theta^{t}\right)\right)\right] \pi^{t}\left(\theta^{t}\right)$ subject to $\forall t, \theta^{t-1}, k<$ $K, \Delta U_{t}\left(\left\{u_{t+s}\left(\theta^{t-1}, \cdot\right)\right\}_{s=0}^{\infty}, \widehat{\theta}_{k}, \widehat{\theta}_{k+1}\right) \geq 0$, where $B_{0}^{t}=\beta^{t} \prod_{s=0}^{t-1}\left(1+\psi_{s}\right)$ and $q_{t}=\widehat{q}_{t} / B_{0}^{t}$.

Then $\left\{\alpha^{\left(\zeta_{0}, \gamma\right)}\right\}$ solves the planner's problem (32) at $\left\{\left\{\widehat{q}_{t}\right\}_{t=0}^{\infty}, \underline{U}, \Phi_{0}, \Psi_{0}\right\}$.

To simplify the analysis, we assume that the $\Psi_{0}$ distribution is degenerate at one, and drop the explicit indexing of variables by $\gamma$. It is natural to focus on stationary allocations that solve the credible insurance problem at an appropriate initial and invariant measure $\Phi_{q}$ and for a sequence of prices with appropriate steady growth rate. In this situation the $\left\{\psi_{t}\right\}$ terms take a constant value $\psi_{q}$ and the optimisation problem in the third part of Proposition 11 above resembles that of a component planner with discount factor $\beta\left(1+\psi_{q}\right)$ in excess of the agent's discount factor $\beta$. We will refer to $\beta\left(1+\psi_{q}\right)$ as the effective discount factor of the planner. More explicitly, we define a stationary credible equilibrium as follows.

Definition 3 A stationary credible equilibrium at $(\underline{U}, q)$ is a tuple $\left(\Phi_{q}, \psi_{q}, V_{q}^{*}, \zeta_{q}^{*}, \eta_{q}^{*}\right)$ satisfying

latter, the Pareto weigthing scheme is given by: $\gamma_{t}\left(\zeta_{0}, \gamma\right)=\gamma+\psi_{t}+\frac{\left(\zeta_{0}-\gamma\right)}{\prod_{s=0}^{t-1}\left(1+\psi_{s}\right)}$. In the former, the weighting scheme lacks the extra $\psi_{t}$ term to the right of the equality. This does not affect the form of the law of motion for endogenous Pareto weights that we derive. 
1.

$$
\begin{aligned}
V_{q}^{*}(\zeta)=\inf _{\eta \in \Lambda(\zeta)} \sum_{\widehat{\theta}_{k} \in \Theta} & \left\{W\left(\left[\zeta+\eta\left(\widehat{\theta}_{k}\right)-\eta\left(\widehat{\theta}_{k-1}\right) \frac{\widehat{\theta}_{k-1}}{\widehat{\theta}_{k}} p_{k-1}+\psi_{q}\right] \widehat{\theta}_{k} ; q\right)\right. \\
& \left.+\beta\left(1+\psi_{q}\right) V_{q}^{*}\left(\frac{1}{1+\psi_{q}}\left[\zeta+\eta\left(\widehat{\theta}_{k}\right)-\eta\left(\widehat{\theta}_{k-1}\right) p_{k-1}\right]+\left(\frac{\psi_{q}}{1+\psi_{q}}\right)\right)\right\} \pi\left(\widehat{\theta}_{k}\right),
\end{aligned}
$$

2. $\eta_{q}^{*}$ attains the solution to (36);

3. $\zeta_{q}^{*}$ satisfies $\zeta_{q}^{*}\left(\zeta, \widehat{\theta}_{k}\right)=\frac{1}{1+\psi}\left[\zeta+\eta_{q}^{*}\left(\zeta, \widehat{\theta}_{k}\right)-\eta_{q}^{*}\left(\zeta, \widehat{\theta}_{k-1}\right) p_{k-1}\right]+\left(\frac{\psi_{q}}{1+\psi_{q}}\right)$;

4. $\Phi_{q}$ is an invariant measure of the Markov process induced by $\zeta_{q}^{*}$ and $\pi$;

5. $\sum_{t=0}^{\infty} \beta^{t}\left(1+\psi_{q}\right)^{t}<\infty ;$ and

6. letting $U(\zeta)$ denote the lifetime utility to an agent with initial Pareto-Negishi weight $\zeta$ under the allocation induced by $\zeta_{q}^{*}$ and $\eta_{q}^{*}, \int_{Z} U(\zeta) \Phi_{q}(d \zeta)=\underline{U}$.

We call the family of utility allocations $\left\{\alpha^{\zeta_{0}}\right\}_{\zeta_{0} \in Z}$ induced by $\zeta_{q}^{*}$ and $\eta_{q}^{*}$ a stationary credible allocation at $(\underline{U}, q)$.

It follows from Proposition 11 and our earlier analysis of recursive problems with high planner discount factors, that a stationary credible allocation at $(\underline{U}, q)$ with invariant measure $\Phi_{q}$ and multiplier $\psi_{q}$ solves the earlier credibility problem (32) at $\left\{\left\{q\left(1+\psi_{q}\right)^{t}\right\}_{t=0}^{\infty}, \underline{U}, \Phi_{q}, 1_{\{1\}}\right\}$. Additionally, we can use our earlier results on optimal stationary allocations from high planner discount economies to establish the existence of a stationary credible allocation. Given a pair $\left(q, \psi_{q}\right)$, under the conditions of Proposition 7, optimal value and policy functions $\left(V_{q}^{*}, \zeta_{q}^{*}, \eta_{q}^{*}\right)$ exist with the latter inducing an invariant distribution $\Phi_{q}$. The tuple $\left(\Phi_{q}, \psi_{q}, V_{q}^{*}, \zeta_{q}^{*}, \eta_{q}^{*}\right)$ is then a stationary credible equilibrium at $\left(q, \int_{Z} U(\zeta) \Phi_{q}(d \zeta)\right)$. Moreover, our earlier results concerning no immiseration and social mobility extend to stationary credible equilibria. For example, when the agent's utility functions are bounded below, the credibility constraints do not rule out the possibility that the planner immiserates a positive 
measure of agents. However, an application of our previous results from high planner discount economies to this setting does. Additionally, when the conditions of Proposition 10 apply, then the policy function $\zeta_{q}^{*}$ from the stationary credible equilibrium yields an ergodic Markov process, with the implications for social mobility and caste systems described earlier.

A stationary credible equilibrium resembles the equilibrium of a Bewley-type economy with $\psi_{q}$ serving as the analogue of an equilibrium price. Consequently, it is relatively straightforward to compute. We provide such numerical calculations in Section 8. We use them to illustrate the equilibrium policy functions and stationary distributions for some sample economies. Specifically, we show how these objects change as $\underline{U}$ and $\psi_{q}$ vary.

\section{Budget constraints}

So far we have treated the pricing sequence $\left\{q_{t}\right\}_{t=0}^{\infty}$ as a parameter. However, if the model is augmented with a resource or budget constraint for the planner, then these prices can be derived endogenously as shadow resource prices. We briefly discuss the issue in the context of a model with an exogenous planner discount factor. ${ }^{17}$ To simplify the exposition, we assume a distribution over $\gamma$ values concentrated at 1 and drop explicit reference to the long run weight $\gamma$ in the notation.

In the remainder of this section, we will refer to an initial Pareto-Negishi weight distribution $\Phi_{0}$ and a family of utility allocations $\left\{\alpha \zeta_{0} \in A\right\}$ as a population allocation. Let $\left\{R_{t}\right\}_{t=0}^{\infty}$ be a fixed sequence of resource quantities and define the period $t$ resource constraint (for population allocations) by:

$$
R_{t} \geq \int_{Z} \sum_{\theta^{t} \in \Theta^{t+1}} C\left(u_{t}^{\zeta_{0}}(\theta)\right) \pi^{t}\left(\theta^{t}\right) \Phi_{0}(d \zeta)
$$

\footnotetext{
${ }^{17}$ The credibility model could be similarly augmented.
} 
The planner's problem with resource or budget constraints can then be written as:

$$
\begin{aligned}
& \sup _{\left\{\alpha \zeta_{0} \in A\right\}} \int_{Z} \sum_{t=0}^{\infty} \lambda^{t} \gamma_{t}\left(\zeta_{0}\right) \sum_{\theta^{t} \in \Theta^{t+1}} \theta_{t} u_{t}^{\zeta_{0}}\left(\theta^{t}\right) \pi^{t}\left(\theta^{t}\right) \Phi_{0}\left(d \zeta_{0}\right) \\
& \text { s.t. } \forall \zeta_{0}, t, \theta^{t-1}, k<K, \quad \lambda^{t} \Delta U_{t}\left(\alpha^{\zeta_{0}}\left(\theta^{t-1}\right), \widehat{\theta}_{k}, \widehat{\theta}_{k+1}\right) \geq 0 \\
& \forall t, \quad R_{t} \geq \int_{Z} \sum_{\theta^{t} \in \Theta^{t+1}} C\left(u_{t}^{\zeta_{0}}(\theta)\right) \pi^{t}\left(\theta^{t}\right) \Phi_{0}(d \zeta) .
\end{aligned}
$$

By a fairly standard argument (e.g. Luenberger (1969)), if a family of allocations $\left\{\alpha^{\zeta_{0}}\right\}$ solves the component planner problems $(11)$ at prices $\left\{q_{t}\right\}_{t=0}^{\infty} \in \ell_{1,+}(\lambda):=\left\{\left\{x_{t}\right\}_{t=0}^{\infty}: \sum_{t=0}^{\infty} \lambda^{t}\left|x_{t}\right|<\infty, \forall t, x_{t} \geq 0\right\}$ and if the population allocation $\left\{\Phi_{0},\left\{\alpha^{\zeta_{0}}\right\}\right\}$ satisfies the resource constraints (37) with equality at all dates, then $\left\{\alpha^{\zeta_{0}}\right\}$ solves (38) subject to these constraints.

In general, it is difficult to characterise the optimal allocation $\left\{\alpha \zeta_{0}\right\}$ and price sequence at a particular sequence of resource quantities $\left\{R_{t}\right\}_{t=0}^{\infty}$ (though, see our discussion of the CRRA, constant resource quantity case below). On the other hand, given a price sequence $\left\{q_{t}\right\}_{t=0}^{\infty} \in \ell_{1,+}(\lambda)$ and initial distribution $\Phi_{0}$ one can first solve for the optimal allocation at these parameters (by solving the earlier component planner problems (11)) and then in a second step, compute the corresponding aggregate resource costs. The allocation then solves (38) when the sequence $\left\{R_{t}\right\}$ is equated with these resource costs. In particular, if $q$ is constant, and the conditions of Proposition 7 hold, then the component planner's optimal policy functions admit an invariant distribution $\Phi_{q}$ over Pareto-Negishi weights. The corresponding constant resource $\operatorname{cost} R_{q}$ is given by:

$$
R_{q}=\int_{Z} \sum_{\widehat{\theta}_{k}} C\left(u^{*}\left(\widehat{\theta}_{k}\left(\zeta+\eta_{q}^{*}\left(\zeta, \widehat{\theta}_{k}\right)-\eta_{q}^{*}\left(\zeta, \widehat{\theta}_{k-1}\right) \frac{\widehat{\theta}_{k-1}}{\widehat{\theta}_{k}}\right) ; q\right)\right) \pi\left(\widehat{\theta}_{k}\right) \Phi_{q}(d \zeta)
$$

where $u^{*}(\rho, q)=\arg \sup _{u \in D} \rho u-q C(u)$. Since $u^{*}(\rho ; q)=\frac{\partial C}{\partial u}^{-1}\left(\frac{\rho}{q}\right)$, we have that $R_{q}$ is finite if $q>0$ and $C \circ C^{\prime-1}$ is concave. In this case, the allocations $\left\{\alpha^{\zeta_{0}}\right\}$ induced by the component planner's optimal policy functions solve the planner's problem (38) when $\Phi_{0}=\Phi_{q}$ and each $R_{t}=R_{q}<\infty$.

Rather more can be said for the CRRA economy. In this case, with constant prices, the component planner's 
value function satisfies:

$$
\begin{aligned}
V_{q}(\zeta)=\inf _{\eta \in \Lambda(\zeta)} \sum_{\widehat{\theta}_{k} \in \Theta}\left\{\frac{\sigma}{1-\sigma}\left(\frac{1}{q}\right)^{\frac{1-\sigma}{\sigma}}\left(\left[\zeta+\eta\left(\widehat{\theta}_{k}\right)-\eta\left(\widehat{\theta}_{k-1}\right) \frac{\widehat{\theta}_{k-1}}{\widehat{\theta}_{k}} p_{k-1}\right] \widehat{\theta}_{k}\right)^{\frac{1}{\sigma}}\right. \\
\left.+\lambda V_{q}\left(\frac{1}{1+\psi}\left[\zeta+\eta\left(\widehat{\theta}_{k}\right)-\eta\left(\widehat{\theta}_{k-1}\right) p_{k-1}\right]+\left(\frac{\psi}{1+\psi}\right)\right)\right\} \pi\left(\widehat{\theta}_{k}\right) .
\end{aligned}
$$

Dividing through by $\left(\frac{1}{q}\right)^{\frac{1-\sigma}{\sigma}}$, we observe that $\left(\frac{1}{q}\right)^{\frac{\sigma-1}{\sigma}} V_{q}(\zeta)=T_{1}\left(\frac{1}{q}\right)^{\frac{\sigma-1}{\sigma}} V_{q}(\zeta)$, where $T_{(q=1)}$ denotes the Bellman operator when $q=1$. Now for $q>0,\left(\frac{1}{q}\right)^{\frac{\sigma-1}{\sigma}} V_{q} \in \mathcal{V} .^{18}$ By arguments similar to those given earlier, when $\sigma>1, T_{(q=1)}$ is a contraction on $\mathcal{V}$ with unique fixed point $V_{(q=1)}$ in $\mathcal{V}$. Hence, $V_{q}=\left(\frac{1}{q}\right)^{\frac{1-\sigma}{\sigma}} V_{(q=1)}$. It follows that the policy functions and stationary distribution over Pareto-Negishi weights are independent of q; $q$ simply scales the utilities and consumptions of agents up or down. In particular, it is easy to check that $R_{q}=\left(\frac{1}{q}\right)^{\frac{1}{\sigma}} R_{(q=1)}$, where $R_{(q=1)}$ is the steady state resource cost when $q=1$. Consequently, once $R_{(q=1)}$ is obtained, it is straightforward to compute the price that would support an arbitrary resource level as part of a stationary solution.

\section{Numerical computations}

In this section, we briefly present some numerical calculations that illustrate and expand upon our previous analysis. In particular, we compute steady state credible equilibria for several economies, focussing on policy functions and steady state distributions. Throughout, CRRA preferences are assumed: $u(c)=c^{1-\sigma} / 1-\sigma$. We fix $\sigma=2$, the agent's discount factor $\beta=0.9$, the shock set $\Theta$ to consist of 4 values evenly distributed between 0.8 and 1.2 and for $k=1, \ldots, 4, \pi\left(\widehat{\theta}_{k}\right)=0.25$. We also fix the aggregate amount of resources used in the stationary credible equilibrum to be 1 . The lower bound on the planner's continuation utility, or, equivalently, the steady state expected utility of agents is allowed to vary across a range. We obtain the corresponding steady

\footnotetext{
${ }^{18}$ The bounding prices $\underline{q}$ and $\bar{q}$ can be set arbitrarily small or large.
} 
state price $q$ and multiplier $\psi_{q}$. The effective discount factor of the planner then varies according to $\beta\left(1+\psi_{q}\right)$.

Solutions to the component planner's dynamic programming problem are computed using value iteration at $q=1$ and a fixed value for $\psi_{q}$. We approximate value functions using Schumaker splines on an interval. These are extended off of the interval when necessary using polynomials that remain within the bounds $\underline{V}$ and $\bar{V}$. We use the approximated policy functions to simulate the Markov process for $\zeta$ and compute the implied steady state moment for resources and agent lifetime utility. Under the CRRA assumption, the $\zeta_{q}^{*}$ policy functions are independent of $q$. This price simply scales up the resources and utility of agents. In a second step, we adjust this price until the aggregate amount of resources used is 1 . We scale the lifetime utility of agents up or down accordingly. This gives us an (approximated) stationary credible equilibrium with aggregate resource cost of 1 . We run through many different values of $\psi_{q}$ and, hence, build up stationary credible equilibria at many values of $\underline{U}$.



Figure 1: $\psi_{q}$ and $\underline{U}$.

Figure 1 begins our analysis by showing that $\psi_{q}$, and, hence, the effective discount factor for the planner, is increasing in the payoff bound $\underline{U}$. Thus, the tighter the credibility constraint, the greater the effective patience of the planner. The autarkic payoff $(-10)$ and corresponding $\psi_{q}$ (slightly above 0.005$)$ are marked with arrows, 
as is the first best, full insurance payoff $(-9.972)$. The largest feasible stationary credible equilibrium payoff is close to, but strictly less than this (about -9.976). ${ }^{19}$ This suggests that the welfare loss associated with the restriction to stationary credible equilibria may be small.

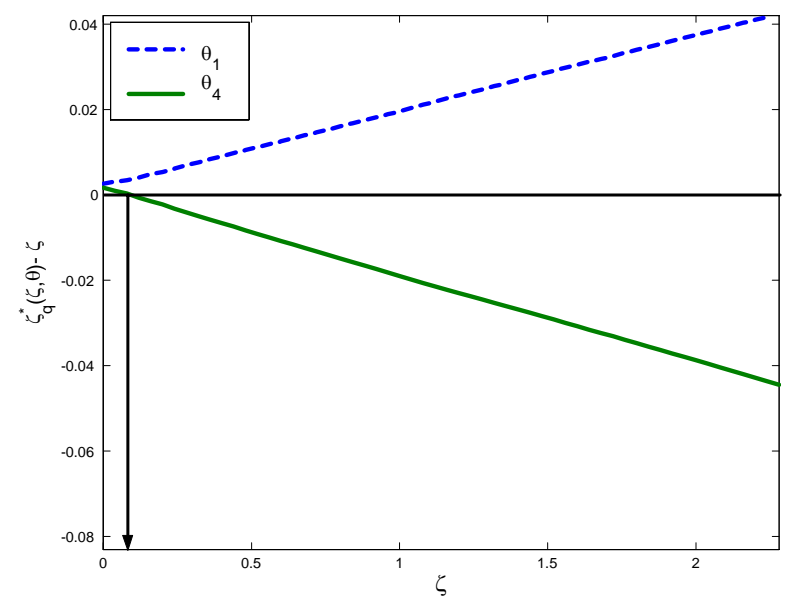

Figure $2 \mathrm{~A}: \zeta_{q}^{*}(\zeta, \theta)-\zeta, \psi_{q}=0.024$

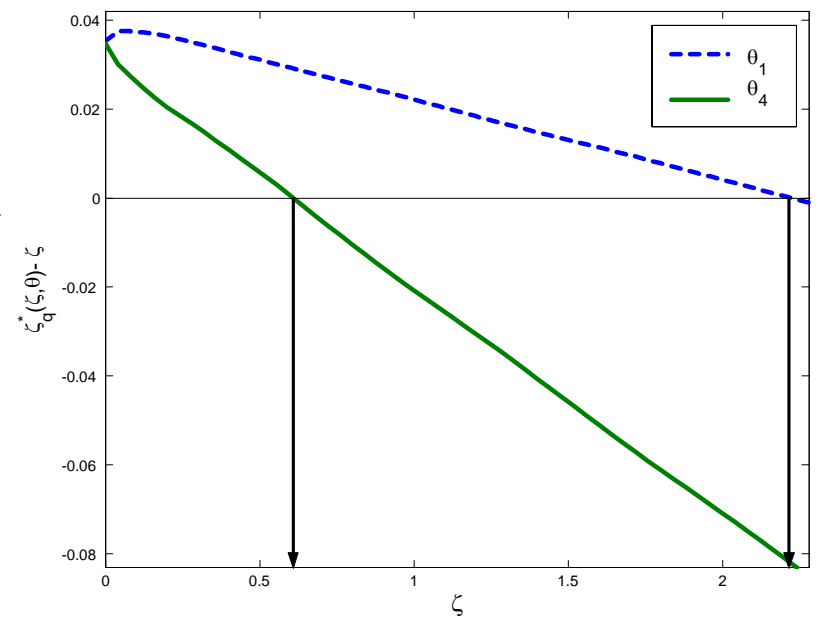

Figure $2 \mathrm{~B}: \zeta_{q}^{*}(\zeta, \theta)-\zeta, \psi_{q}=0.035$

We now turn to agent policy functions. Figures $2 \mathrm{~A}$ and $2 \mathrm{~B}$ show $\Delta \zeta(\zeta, \theta):=\zeta_{q}^{*}(\zeta, \theta)-\zeta$, for $\theta=\left\{\widehat{\theta}_{1}, \widehat{\theta}_{4}\right\}$. Thus, they show the change in an agent's Pareto-Negishi weight contingent on the realisation of the lowest and highest shocks. Figure $2 \mathrm{~A}$ shows what happens when $\psi_{q}=0.0024$ (and $\underline{U}=-10.03$ ). In this case, the $\Delta \zeta(\zeta, \theta)$ functions diverge from a positive value at $\zeta=0$. The lower policy function, $\Delta \zeta\left(\zeta, \widehat{\theta}_{4}\right)$, associated with the high shock, crosses the $\zeta$-axis at $\zeta$ value slightly below 0.1 . Let $\underline{\zeta}$ denote this value, then it is immediate from the figure that the interval $[0, \underline{\zeta}]$ is non-absorbing. In fact, the $\zeta_{q}^{*}\left(\cdot, \widehat{\theta}_{k}\right)$ policy functions are monotone, so that this set is transient; after a finite number of periods, an agent's Pareto-Negishi weight is almost surely above $\underline{\zeta}$. Thereafter, it remains above $\underline{\zeta}$. Clearly, agents are not immiserated. Since the $\zeta_{q}^{*}\left(\cdot, \widehat{\theta}_{k}\right)$ functions are monotone, the associated Markov process is strongly ergodic, indicating social mobility and an absence of multiple caste

\footnotetext{
${ }^{19}$ To place this into perspective, first best consumption needs to be reduced by about $0.05 \%$ to achieve this lower payoff.
} 
systems. However, the support of the invariant measure is large, it is the whole region $[\underline{\zeta}, \infty)$, and there is always a positive, though decreasing, mass of agents above any finite $\zeta$. In this sense, substantial inequality remains.

Figure 2B plots the $\Delta \zeta(\zeta, \theta)$ for the much higher $\psi_{q}$ value 0.035 and a correspondingly higher $\underline{U}$ value (of -9.977). Thus, this is an economy with a tighter credibility constraint and a higher effective discount factor for the planner. Now, $\Delta \zeta\left(\zeta, \widehat{\theta}_{4}\right)$ crosses the $\zeta$-axis at a higher value of $\underline{\zeta}=0.6$. Again, $\zeta_{q}^{*}$ is monotone and $[0, \underline{\zeta}]$ is transient. Evidently, this lower transient region is larger than before. In addition, the upper function $\Delta \zeta\left(\zeta, \widehat{\theta}_{1}\right)$ crosses zero from above at approximately 2.42 , indicating that the region above $\zeta=2.42$ is transient as well. Thus, an agent's Pareto-Negishi weight is almost surely absorbed by the set [0.6,2.42]. This and other numerical examples indicate that as $\underline{U}$ and $\psi_{q}$ rise, the support of the invariant measure from the corresponding Pareto-Negishi weight Markov process shrinks. By way of contrast, for the case $\psi_{q}=0$ (not shown), the functions $\Delta \zeta(\zeta, \theta)$ diverge from 0 as $\zeta$ increases above 0 . By the immiseration result, agents' Pareto-Negishi weights are almost surely absorbed by 0 .

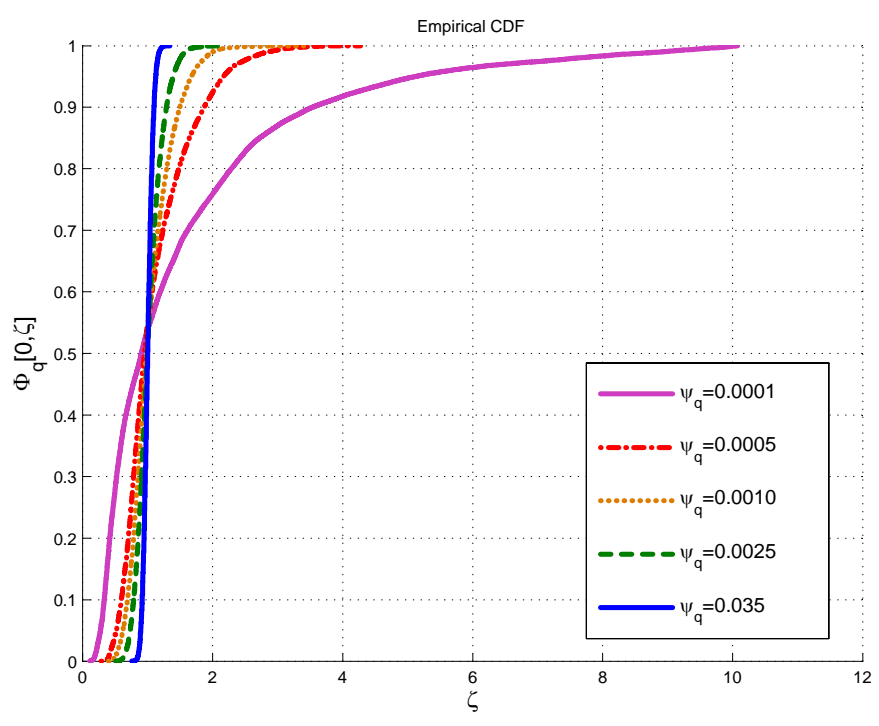

Figure 3: CDFs

The monotonicity of the computed policy functions indicates that the underlying constant price economies 
exhibit an ergodic Markov process for $\zeta$ weights, and, consequently, admit a unique invariant probability distribution $\Phi_{q}$. In Figure 3, we illustrate the cumulative distribution functions $F(\zeta)=\Phi_{q}([0, \zeta])$ implied by $\Phi_{q}$ for a variety of $\psi_{q}$ values. The corresponding $\underline{U}$ values can be obtained from Figure 1 . The lowest value of $\psi_{q}$, equal to $1 \times 10^{-4}$, implies considerable inequality with non-negligible probability mass across a wide range of $\zeta$ values. The highest value of $\psi_{q}, 0.035$, implies a distribution concentrated in a fairly narrow interval of 1 . The invariant distributions at values of $\psi_{q}$ between $1 \times 10^{-4}$ and 0.035 are intermediate between these cases.

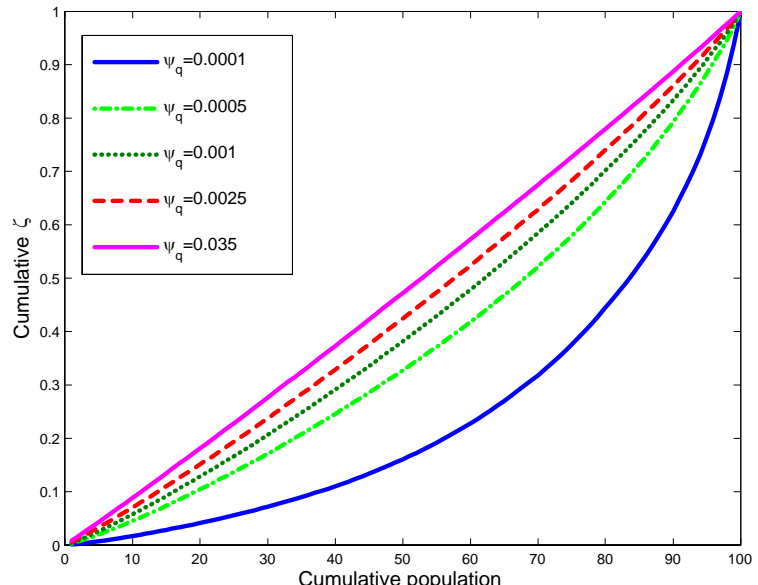

Figure 4A: Lorenz curve, $\zeta$

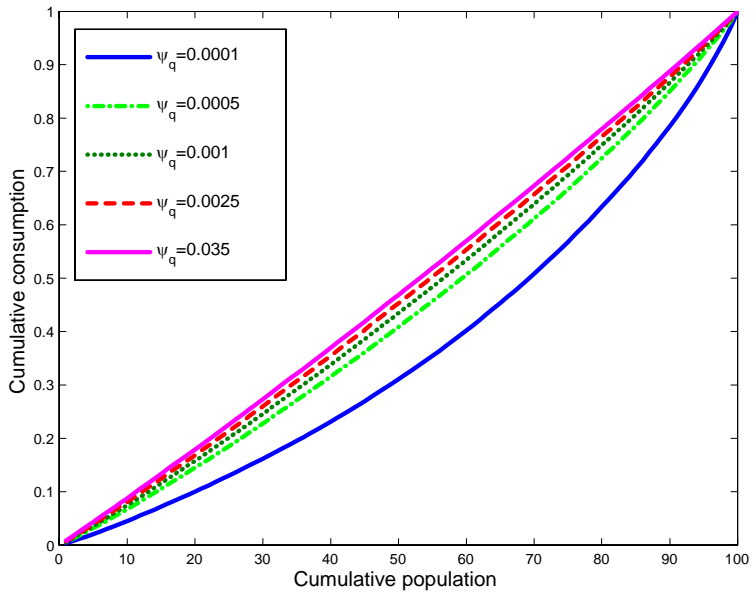

Figure 4B: Lorenz curve, consumption

Figure $4 \mathrm{~A}$ shows the corresponding family of Lorenz curves for the $\zeta$-distribution; each curve is drawn for a different value of $\psi_{q}$. These curves also indicate that inequality is falling in $\psi_{q}$ and $\beta\left(1+\psi_{q}\right){ }^{20}$ Moreover, they suggest that the degree of inequality is highly non-linear in $\psi_{q}$. Inequality is extreme when $\psi_{q}=0$, with a measure zero set of agents having an infinite $\zeta$ weight and consuming all resources in the economy, while the rest have zero $\zeta$ weight and no consumption. ${ }^{21}$ When $\psi_{q}=1 \times 10^{-4}$, inequality is still large. However, it falls rapidly as $\psi_{q}$ rises. The Lorenz curves for consumption, Figure 4B, have a qualitatively similar form.

\footnotetext{
${ }^{20}$ Recall that a Lorenz curve coincident with the 45 degree line is associated with full equality. A convex Lorenz curve that "bows" towards the axes indicates more inequality.

${ }^{21}$ In this case, the $\zeta$ Lorenz curve coincides with the lower and right hand axes of Figure $4 \mathrm{~A}$.
} 
We conclude this section by graphing the Gini coefficients for $\zeta$ as a function of $\psi_{q}$ and $\underline{U}$. These graphs show that the Gini coefficient converges towards 1 (extreme inequality) as $\psi_{q}$ converges towards 0 or $\underline{U}$ converges towards $-\infty$. The Gini coefficient is monotone decreasing and non-linear in each of these variables.

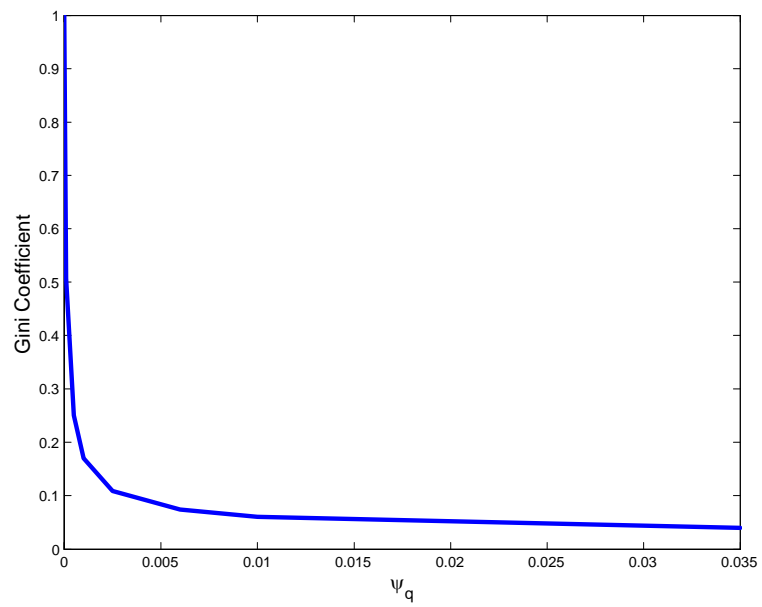

Figure 5A: Gini coefficient, $\zeta$



Figure 5B: Gini coefficient for $\zeta$

Overall, the numerical calculations enhance our earlier theoretical results by confirming that the tighter the credibility constraint, the higher is the effective discount factor and the lower the degree of inequality in the corresponding stationary credible equilibrium. Moreover, the existence of stationary credible equilibria with planner (expected agent) continuation payoffs close to the first best is significant. It suggests that while the imposition of credibility constraints must imply some welfare loss, this welfare loss may be small. Thus, the agent (or dynasty) may not have to give up a great deal to avoid long run immiseration.

\section{Conclusion}

The analysis in this paper implies that the immiseration result is a pathology of environments with equal planner and agent discount factors. In environments in which the planner's discount rate exceeds that of the agents, a stationary distribution over utilities and consumptions often emerges. Relatedly, immiseration assumes that the 
planner (or government) is free to treat future generations arbitrarily badly in the interests of current generations. As we have argued, at a practical level this seems implausible. Optimal allocations in economies in which such treatment is not possible resemble those in ones with a high planner discount factor. Since allocations are only credible if they are consistent with the feasible long run treatment of agents, credibility restrictions imply social patience, or, at least, patient-like behaviour.

At a technical level this paper, shows the value of the Marcet-Marimon procedure for the analysis of (concave) dynamic moral hazard problems. It also applies new techniques from Markov process theory to the study of such problems. These technical contributions should be useful in settings beyond the one considered in the paper.

Our social credibility constraints seek to capture the ability of groups of agents to resist ex post reductions in their continuation utility. This ability is captured by an agent specific weight and by a lower bound on the weighted sum of agent utilities. The weights and the bound are parameters. We think it would be extremely interesting to endogenise these parameters in the context of explicit policy or political economy games. In Sleet and Yeltekin (2004), we consider one such game in which the planner or government cannot commit. But many other possibilities exist. We leave this important area of extension to future work.

\section{References}

[1] Abreu, D. 1988. On infinitely repeated games with discounting. Econometrica 56:383-396.

[2] Atkeson, A. and R. Lucas. 1992. On efficient distribution with private information. Review of Economic Studies 59:427-453.

[3] Atkeson, A. and R. Lucas. 1995. Efficiency and equality in a simple model of efficient unemployment insurance. Journal of Economic Theory 66:64-88.

[4] Chari, V.V. and P. Kehoe. 1990. Sustainable plans. Journal of Political Economy 98:617-636. 
[5] Farhi, E. And I. Werning. 2005. Inequality, social discounting and estate taxation. Working paper.

[6] Green, E.. 1987. Lending and the smoothing of uninsurable income. In E. Prescott and N. Wallace (eds.) Contractual arrangements for intertemporal trade. University of Minnesota Press: Minneapolis.

[7] Hernández-Lerma, O. and J. Lasserre. 1999. Further topics on discrete-time Markov control processes. Springer-Verlag: New York.

[8] JudD, K. 1985. The law of large numbers with a continuum of IID random variables. Journal of Economic Theory 35:19-25.

[9] Lasota, A. M. Mackay. 1994. Chaos, fractals and noise. Springer-Verlag: Berlin.

[10] Luenberger, D. 1969. Optimization by vector space methods. Wiley.

[11] Marcet, A. and R. Marimon. 1999. Recursive contracts. Working paper, UPF.

[12] Messner, M. and N. Pavoni. 2004. A note on the recursive saddle point method. Working paper.

[13] Phelan, C. 1995. Repeated moral hazard and one-sided commitment. Journal of Economic Theory 66:488506.

[14] Phelan, C. 1998. On the long run implications of repeated moral hazard. Journal of Economic Theory 79:174-191.

[15] Phelan, C. 2003. Opportunity and social mobility. Staff Report 323, FRB Minneapolis.

[16] Sleet, C. And Ş. Yeltekin. 2004. Credible social insurance. Working paper.

[17] Thomas, J. and T. Worrall. 1990. Income fluctuation and asymmetric information: an example of a repeated principal-agent problem. Journal of Economic Theory 51:367-390. 